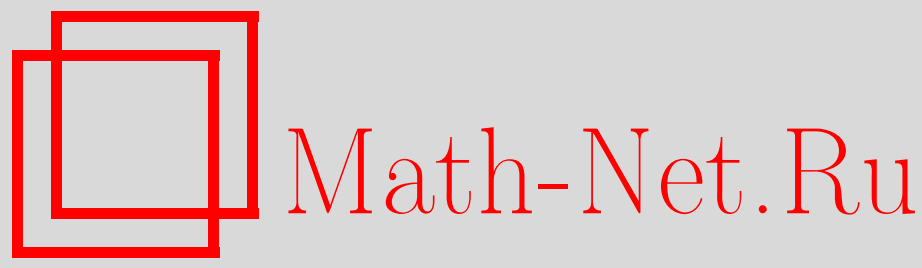

А. Ю. Морозов, Унитарные интегралы и связанные с ними матричные модели, ТМФ, 2010, том 162, номер 1, 340

DOI: https://doi.org/10.4213/tmf6453

Использование Общероссийского математического портала Math-Net.Ru подразумевает, что вы прочитали и согласны с пользовательским соглашением http://www . mathnet.ru/rus/agreement

Параметры загрузки:

IP : 34.227 .88 .159

26 апреля 2023 г., 08:38:59

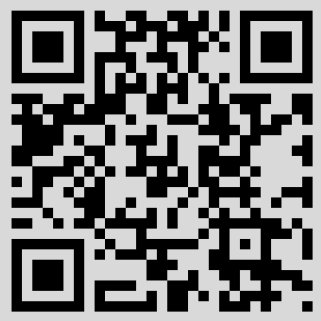




\section{УНИТАРНЫЕ ИНТЕГРАЛЫ И СВЯЗАННЫЕ С НИМИ МАТРИЧНЫЕ МОДЕЛИ}

Дан краткий обзор основных свойств интегралов по унитарным матрицам. Эти интегралы анализируются с помощью трех матричных моделей: обычной унитарной модели, модели Гросса-Брезана-Виттена и модели Хариш-ЧандрыИциксона-Зюбера. Особое внимание уделено нетривиальным аспектам теории, от аномалии 'т Хоофта-де Вита в унитарных интегралах до проблемы вычисления корреляторов с мерой Ициксона-Зюбера. Из технических средств особо отмечен метод разложения по характерам. Унитарные интегралы все еще недостаточно исследованы, и с ростом внимания к этой области теории следует ожидать множества новых результатов.

Ключевые слова: матричная модель, унитарная группа.

\section{1. ВВЕДЕНИЕ}

Интегралы по неэрмитовым матрицам на протяжении всей истории матричных моделей пользовались значительно меньшим вниманием, чем интегралы по эрмитовым матрицам [1]-[27]. Это не вполне справедливо: неэрмитовы модели не менее важны в приложениях, чем эрмитовы, их можно успешно исследовать теми же методами, и они естественным образом включаются в объединенную М-теорию матричных моделей [16]. Тем не менее при недавней реинкарнации матричных моделей [6]-[18] неэрмитовым моделям снова не досталось должного внимания - серьезному анализу не подвергнуты ни их нетривиальная фазовая структура в духе работ [6], [11], ни теоретико-полевая переформулировка в духе статей [12], ни комбинаторное описание гауссовой (с одним разрезом) фазы в духе работы [18] несмотря на то, что все эти проблемы заведомо находятся в рамках применимости развиваемых методов.

Настоящий текст тоже слишком короток для того, чтобы подробно описать пути решения этих важных вопросов. Вместо этого мы сконцентрируем свое внимание на дополнении к этим универсальным предметам исследования: методах и результатах, которые специфичны для одной конкретной - и самой важной из всех неэрмитовых моделей - теории гауссовых унитарных ансамблей. Важность унитарных

* Институт теоретической и экспериментальной физики, Москва, Россия. E-mail: morozov@itep.ru 
интегралов очевидна уже с точки зрения теории Янга-Миллса: они описывают угловые (цветовые) степени свободы, являющиеся дополнением к диагональным (бесцветным или адронным) компонентам, которые адекватно описываются эрмитовыми моделями [4] и их “дуальными” партнерами из семейства обобщенных моделей Концевича (OMK) [4], [21], [22]. Конечно, эта дополнительность не означает, что к задаче об унитарных интегралах неприменимы различные методы, использующие собственные значения матриц, от техники ортогональных полиномов [1] до решения условий Вирасоро [23] итерациями [24], [11] или с помощью квантовой теории поля [12]. Все эти методы могут применяться, и, как всегда, они весьма эффективны и плодотворны (см. работы [25], [26]). Дополнительность проявляется в другом: наибольший интерес представляют вопросы иного типа, относящиеся к корреляторам недиагоналъных матричных элементов, а не следов или детерминантов.

Мощную и частично адекватную технику исследования таких аспектов унитарных интералов предоставляет теория разложений по характерам. Одна из основных интриг при этом связана с относительной ролью и взаимоотношением групп двух различных типов: линейных $(G L)$ и симметрических $(S)$. В настоящее время эти взаимоотношения привлекают возрастающее внимание из-за тесной связи между теорией Янга-Миллса, с одной стороны, и наилучшим образом представленной в контексте матричных теорий моделью Казакова-Мигдала [27]-[33] и описывающей разветвленные накрытия римановых поверхностей [34]-[40] теорией Гурвица, которая связана с производящими функциями Гурвица-Концевича [35]-[38] и довольно необычными матричными моделями [36], [37], с другой стороны.

Настоящая статья представляет собой не более чем краткое введение в эти аспекты теории унитарных интегралов. Наше изложение будет сосредототочено вокруг трех основных унитарных матричных моделей:

- исходной унитарной модели [25], предназначенной для описания корреляторов следов унитарных матриц стандартными матрично-модельными методами (по этой причине мы уделим этой теории не слишком много внимания);

- модели Брезана-Гросса-Виттена (БГВ) [41], описывающей корреляторы произвольных матричных элементов (а не только следов); эта модель была включена, в тот момент довольно неожиданно, в семейство OMK в работах [32], [33] и в конце концов нашла свое значимое место в М-теории матричных моделей [16] в статье [42];

- модели Ициксона-Зюбера (ИЗ) [43], [44] - упрощенной одноплакетной версии теории Казакова-Мигдала [27], которая тоже описывает произвольные корреляторы, только усредненные с нетривиальным, но точно решаемым весом (действием ИЗ), и является давним и сложным предметом исследования [29]-[31], недавно вновь привлекшим к себе внимание в работе [14].

В этой области, которая в отличие от теории эрмитовых интегралов находится на весьма ранней ступени развития, ожидается множество новых и значительно более глубоких результатов, возможно, уже в самом близком будущем. Из-за этого содержание настоящей статьи поневоле неполно и имеет лишь вре́менную ценность все это не более чем предварительное введение в предмет, который еще до конца не оформился. Тем не менее мы попытались сконцентрироваться на фактах и фор- 
мулах, которые имеют непреходящее значение и серьезные шансы остаться в числе основополагающих, даже когда вся область приобретет черты завершенности и самосогласованности. Напротив, меньше внимания уделено сиюминутно важным аспектам теории, которые заведомо будут существенно улучшены и переформулированы в иных, пока неизвестных терминах. Некоторые из этих вопросов просто опущены, чтобы избежать излишней перегрузки текста.

\section{2. УНИТАРНЫЕ ИНТЕГРАЛЫ И МОДЕЛЬ БГВ}

2.1. Мера. Унитарные интегралы - это интегралы по унитарным матрицам $U$ размера $N \times N$, удовлетворяющим нелинейным условиям $U U^{\dagger}=U^{\dagger} U=I$. Интегралы берутся по инвариантной мере Хаара $[d U]$. В отличие от случая эрмитовых матриц $H=H^{\dagger}$, где инвариантная мера - это просто $d H=\bigwedge_{i, j} d H_{i j}$, унитарная мера Хаара $[d U]$ нетривиальна и нелинейна по $U$. Если унитарные матрицы выражены в терминах эрмитовых, то меру $[d U]$ тоже можно выразить через $d H$, например

$$
\begin{aligned}
& U=e^{i H} \Longrightarrow\|\delta U\|^{2}=\operatorname{tr}\left(U^{\dagger} \delta U U^{\dagger} \delta U\right)=\operatorname{tr}(\delta H)^{2} \Longrightarrow[d U]=d H, \\
& U=\frac{I+i H}{I-i H} \Longrightarrow\|\delta U\|^{2}=4 \operatorname{tr}\left(\frac{I}{I+i H} \delta H \frac{I}{I-i H} \delta H\right) \Longrightarrow[d U] \sim \frac{d H}{\operatorname{det}^{N}\left(1+H^{2}\right)}
\end{aligned}
$$

и аналогично для любых иных мыслимых параметризаций.

В качестве альтернативного подхода можно наложить условие унитарности на произвольную комплексную матрицу $M$ [32], [33], [14]:

$$
[d U]=\delta\left(M M^{\dagger}-I\right) d^{2} M=d^{2} M \int e^{i \operatorname{tr} S\left(M M^{\dagger}-I\right)} d S,
$$

где $S$ - вспомогательная эрмитова матрица, введенная как множитель Лагранжа. Ниже мы будем часто включать множитель $i$ в $S$, что превращает эту матрицу в антиэрмитову, т.е. изменяет контур интегрирования по $S$. Более того, часто можно поглотить, переопределяя $S$, целый нетривиальный матричный множитель, что полностью разрушает эрмитовость, тем не менее и это является зачастую не более чем невинной заменой контура интегрирования.

В дальнейшем мы нормируем меру $[d U]$ так, что $\int[d U]=1$, т.е. делим $[d U]$ на объем унитарной группы $V_{N}$. Этот объем конечен, и его можно вычислить, например, используя ортогональные полиномы (см. раздел 3.9 в первом из обзоров в ссылке [4]). Формула для объема довольно сложная:

$$
V_{N}=\frac{(2 \pi)^{N(N+1) / 2}}{\prod_{k=1}^{N} k !} .
$$

Мы также предполагаем, что множитель $V_{N}^{-1}$ включен в определение эрмитовых матричных интегралов.

2.2. Элементарные унитарные корреляторы в фундаментальном представлении. Имея такое соглашение о нормировках и пользуясь свойствами $S U(N)$-симметрии, мы немедленно получаем

$$
\left\langle U_{i j} U_{k l}^{\dagger}\right\rangle=\int U_{i j} U_{k l}^{\dagger}[d U]=\frac{1}{N} \delta_{i l} \delta_{j k},
$$




$$
\begin{aligned}
& \int U_{i j} U_{i^{\prime} j^{\prime}} U_{k l}^{\dagger} U_{k^{\prime} l^{\prime}}^{\dagger}[d U]= \\
& \quad=\frac{N^{2}\left(\delta_{i l} \delta_{i^{\prime} l^{\prime}} \delta_{j k} \delta_{j^{\prime} k^{\prime}}+\delta_{i l^{\prime}} \delta_{i^{\prime} l} \delta_{j k^{\prime}} \delta_{j^{\prime} k}\right)-N\left(\delta_{i l^{\prime}} \delta_{i^{\prime} l} \delta_{j k} \delta_{j^{\prime} k^{\prime}}+\delta_{i l} \delta_{i^{\prime} l^{\prime}} \delta_{j k^{\prime}} \delta_{j^{\prime} k}\right)}{N^{2}\left(N^{2}-1\right)} .
\end{aligned}
$$

Коэффициенты в правых частях этих формул легко найти, сворачивая друг с другом разные индексы и пользуясь слева условием унитарности. Уже этого примера достаточно, чтобы убедиться, что корреляторы быстро усложняются с ростом числа матриц, и для их вычисления требуется какой-то систематический подход. Такой подход предоставляется теорией модели БГВ из работы [33].

Прежде чем мы займемся этим предметом в п. 2.4, заметим, что корреляторы резко упрощаются, когда мы сворачиваем индексы, т.е. рассматриваем корреляторы следов, а не отдельных матричных элементов. В самом деле, из (4) и (5) мы немедленно получаем, что

$$
\begin{aligned}
\int \operatorname{tr} U \operatorname{tr} U^{\dagger}[d U] & =1, & & \int \operatorname{tr} U^{2} \operatorname{tr}\left(U^{\dagger}\right)^{2}[d U]=2-\delta_{N 1}, \\
\int \operatorname{tr} U^{2}\left(\operatorname{tr} U^{\dagger}\right)^{2}[d U] & =\delta_{N 1}, & & \int(\operatorname{tr} U)^{2}\left(\operatorname{tr} U^{\dagger}\right)^{2}[d U]=2-\delta_{N 1}
\end{aligned}
$$

и т.д. (случай $N=1$ в этих формулах требует отдельного анализа, см.ниже). Эти более простые бесцветные корреляторы изучаются в рамках обычной унитарной модели [26].

Отметим также, что уже в (5) видна нетривиальная зависимость унитарных корреляторов от $N$ с фиктивными полюсами при некоторых целых значениях $N$. Это явление известно как аномалия 'т Хоофта-де Вита [45]. В корреляторах бесцветных величин (6) данные полюсы превращаются в специфические несингулярные поправки при тех же значениях $N$. В (5) этим специальным значением является $N=1$, но чем больше матриц в корреляторе, тем больше диапазон затрагиваемых $N$.

2.3. Из теории унитарной модели. Мы опираемся в данном пункте на результаты работы [26].

Статистическая сумма ${ }^{1)}$ унитарной матричной модели определена как производящая функция корреляторов бесцветных операторов (следов):

$$
Z_{U}(t)=\int \exp \left(\sum_{k=-\infty}^{\infty} t_{k} \operatorname{tr} U^{k}\right)[d U]
$$

Ранее чем в работе [26] данная статистическая сумма изучалась также в работах [25]. Заметим, что $U^{\dagger}=U^{-1}$, так что матрицы, сопряженные степеням $U$, представлены здесь отрицательными степенями $U$. Условия Вирасоро в этой модели имеют вид [26]

$$
\left\{\sum_{k=-\infty}^{\infty} k t_{k}\left(\frac{\partial}{\partial t_{k+n}}-\frac{\partial}{\partial t_{k-n}}\right)+\sum_{1 \leqslant k \leqslant n}\left(\frac{\partial^{2}}{\partial t_{k} \partial t_{n-k}}+\frac{\partial^{2}}{\partial t_{-k} \partial t_{k-n}}\right)\right\} Z_{U}(t)=0, \quad n \geqslant 1
$$

\footnotetext{
1) Мы используем термин "статистическая сумма" как аналог английского "partition function". Важно что это именно сама сумма, а не ее логарифм, для которого употребляется термин "свободная энергия".
} 


$$
\left\{\sum_{k=-\infty}^{\infty} k t_{k}\left(\frac{\partial}{\partial t_{k+n}}+\frac{\partial}{\partial t_{k-n}}\right)+\sum_{1 \leqslant k \leqslant n}\left(\frac{\partial^{2}}{\partial t_{k} \partial t_{n-k}}-\frac{\partial^{2}}{\partial t_{-k} \partial t_{k-n}}\right)\right\} Z_{U}(t)=0, \quad n \geqslant 0 .
$$

Применение условий Вирасоро в этой модели немного необычно: здесь отсутствует сдвиг какой-либо временно́й переменной, и решение представимо в виде формального ряда непосредственно по степеням $t$, а не их отношений к какому-либо фоновому значению одной из переменных; выбор этой переменной определяет выбор одной из многих различных фаз, скажем, в эрмитовой модели [9]. В данном случае аналог $L_{0}$-условия гласит, что

$$
\sum_{k=-\infty}^{\infty} k t_{k} \frac{\partial Z_{U}}{\partial t_{k}}=0
$$

и требует, чтобы мономиальные слагаемые в выражении для $Z_{U}$ имели вид $\prod_{k} t_{k}^{n_{k}}$ с $\sum_{k \geqslant 1} k n_{k}=\sum_{k \geqslant 1} k n_{-k}$, т.е. разрешенными являются слагаемые вида $t_{k} t_{-k}, t_{k} t_{l} t_{-k-l}$ и т.п., но не $t_{k}$ или $t_{k} t_{-k-l}$ с $l \neq 0$. При таком правиле отбора первые члены ряда для $Z_{U}$ суть

$$
Z_{U}=e^{N t_{0}}\left(1+a_{1 \mid 1} t_{1} t_{-1}+a_{2 \mid 2} t_{2} t_{-2}+a_{11 \mid 2}\left(t_{1}^{2} t_{-2}+t_{-1}^{2} t_{2}\right)+a_{11 \mid 11} t_{1}^{2} t_{-1}^{2}+\cdots\right) .
$$

Они описывают корреляторы (6) и полностью определяются низшими условиями Вирасоро с $n= \pm 1, \pm 2$, но несколько необычным образом:

$$
\begin{aligned}
0= & e^{-N t_{0}}\left(\sum_{k=-\infty}^{\infty} k t_{k} \frac{\partial}{\partial t_{k+1}}+\frac{\partial^{2}}{\partial t_{0} \partial t_{1}}\right) Z_{U}= \\
= & t_{-1}\left(-N+N \alpha_{1 \mid 1}\right)+t_{1} t_{-2}\left(-2 \alpha_{1 \mid 1}+\alpha_{2 \mid 2}+2 N \alpha_{2 \mid 11}\right)+ \\
& +t_{1} t_{-1}^{2}\left(-N \alpha_{1 \mid 1}+\alpha_{2 \mid 11}+2 N \alpha_{11 \mid 11}\right)+\cdots .
\end{aligned}
$$

Аналогично

$$
\begin{aligned}
0 & =e^{-N t_{0}}\left(\sum_{k=-\infty}^{\infty} k t_{k} \frac{\partial}{\partial t_{k+2}}+\frac{\partial^{2}}{\partial t_{0} \partial t_{2}}+\frac{\partial^{2}}{\partial t_{1}^{2}}\right) Z_{U}= \\
& =t_{-2}\left(-2 N+N \alpha_{2 \mid 2}+2 \alpha_{2 \mid 11}\right)+t_{-1}^{2}\left(-\alpha_{1 \mid 1}+N \alpha_{2 \mid 11}+2 \alpha_{11 \mid 11}\right)+\cdots
\end{aligned}
$$

Условия Вирасоро требуют, чтобы каждая скобка в правой части обращалась в нуль, и из этого следует, что

$$
\begin{array}{rll} 
& \alpha_{1 \mid 1}=1, \\
\left(N^{2}-1\right) \alpha_{2 \mid 2}=2\left(N^{2}-\alpha_{1 \mid 1}\right)=2\left(N^{2}-1\right) & \Rightarrow \quad \alpha_{2 \mid 2}=2-s \delta_{N, 1}, \\
\left(N^{2}-1\right) \alpha_{2 \mid 11}=0 & \Rightarrow \quad \alpha_{2 \mid 11}=s \delta_{N, 1}, \\
2\left(N^{2}-1\right) \alpha_{11 \mid 11}=\left(N^{2}-1\right) \alpha_{1 \mid 1}=N^{2}-1 & \Rightarrow \quad & \alpha_{11 \mid 11}=\frac{1}{4}\left(2-s \delta_{N, 1}\right),
\end{array}
$$

Таким образом, условия Вирасоро не определяют коэффициенты $\alpha_{2 \mid 2}, \alpha_{2 \mid 11}$ и $\alpha_{11 \mid 11}$ полностью - остается неопределенность при $N=1$. На самом деле все три коэффициента зависят от одного неопределенного параметра $s$, который может быть 
зафиксирован прямым вычислением корреляторов (6) при $N=1$. Подобным образом коэффициенты $\alpha$ с более высоким уровнем градуировки $l=\sum_{k \geqslant 0} k n_{k}>2$ остаются не до конца зафиксированными условиями Вирасоро при $N=1, \ldots, l$. Соответствующие свободные параметры являются аналогами свободных модулей в теории эрмитовых моделей [9], конкретным интегралам по унитарным матрицам отвечает конкретный выбор этих параметров (точка в пространстве модулей).

2.4. Из теории модели БГВ. Статистическая сумма модели БГВ [41] - это производящая функция для всех унитарных корреляторов:

$$
\begin{aligned}
Z_{\mathrm{BGW}} & =\int e^{\operatorname{tr}\left(J^{\dagger} U+J U^{\dagger}\right)}[d U]=\iint e^{i S\left(M M^{\dagger}-I\right)} e^{\left(\operatorname{tr} J^{\dagger} M+J M^{\dagger}\right)} d^{2} M d S \stackrel{(2)}{=} \\
& \stackrel{(2)}{=} \int e^{\operatorname{tr}\left(J J^{\dagger} / S\right)-\operatorname{tr} S} \frac{d S}{\operatorname{det} S^{N}} \stackrel{[S \rightarrow 1 / S]}{=} \int e^{\operatorname{tr}\left(J J^{\dagger} S\right)-\operatorname{tr}(1 / S)} \frac{d S}{\operatorname{det} S^{N}} .
\end{aligned}
$$

Очевидно, что $Z_{\mathrm{BGW}}$ зависит только от собственных значений матрицы $J J^{\dagger}$, т.е. от временны́х переменных $t_{k}=k^{-1} \operatorname{tr}\left(J J^{\dagger}\right)^{k}$.

Модель имеет две кардинально различающиеся фазы, названные в работе [33] фазой Концевича и фазой характеров. Здесь мы рассмотрим только вторую из них, в которой $Z_{\mathrm{BGW}}$ является формальным рядом по положительным степеням $t_{k}$. Условия Вирасоро в этой фазе выводятся обычным образом - как тождества Уорда в духе работ [23], но в этом конкретном случае их можно получить и иначе - как прямое следствие тождества $U U^{\dagger}=I$ для унитарных матриц, выраженного в терминах переменных $t$. Из этого тождества следует, что

$$
0=\left(\frac{\partial}{\partial \tilde{J}^{\dagger}} \frac{\partial}{\partial J}-I\right) Z_{\mathrm{BGW}}=\sum_{n=1}^{\infty}\left(J J^{\dagger}\right)^{n-1} \hat{L}_{n} Z_{\mathrm{BGW}}-I Z_{\mathrm{BGW}}
$$

T.e.

$$
\hat{L}_{n} Z_{\mathrm{BGW}}=\left(\sum_{k=1}^{\infty} k t_{k} \frac{\partial}{\partial t_{k+n}}+\sum_{\substack{a+b=n, a, b \geqslant 0}} \frac{\partial^{2}}{\partial t_{a} \partial t_{b}}\right) Z_{\mathrm{BGW}}(t)=\delta_{n, 1}, \quad n \geqslant 1
$$

что выглядит, как обычные дискретные условия Вирасоро [19], хорошо знакомые из теории эрмитовой модели, только с $n \geqslant 1$ вместо $n \geqslant-1$ и со слегка измененной правой частью при $n=1$.

Поставляя анзац в виде формального ряда для $Z_{\mathrm{BGW}}(t)$,

$$
Z_{N}^{+}(t)=1+\sum_{M \geqslant 1}\left(\sum_{k_{1} \geqslant \cdots \geqslant k_{M} \geqslant 0} c_{N}\left\{k_{a}\right\} \frac{k_{1} t_{k_{1}} \ldots k_{M} t_{k_{M}}}{\left(k_{1}+\cdots+k_{M}\right) !}\right),
$$

в эти условия Вирасоро, можно итеративно вычислить любой заданный коэффициент (см. п. 3.2.1 статьи [33]):

$$
c_{N}\left\{k_{a}\right\}=\hat{c}_{N}\left\{k_{a}\right\} \prod_{l=0}^{k_{1}+\cdots+k_{M}-1} \frac{1}{N^{2}-l^{2}}
$$


где

$$
\begin{gathered}
\hat{c}_{N}(1)=N, \\
\hat{c}_{N}(2)=-N, \quad \hat{c}_{N}(1,1)=N^{2}, \\
\hat{c}_{N}(3)=4 N, \quad \hat{c}_{N}(2,1)=-3 N^{2}, \quad \hat{c}_{N}(1,1,1)=N\left(N^{2}-2\right), \\
\hat{c}_{N}(4)=-30 N, \quad \hat{c}_{N}(3,1)=8\left(2 N^{2}-1\right), \quad \hat{c}_{N}(2,2)=3\left(N^{2}+6\right), \\
\hat{c}_{N}(2,1,1)=-6 N\left(N^{2}-4\right), \quad \hat{c}_{N}(1,1,1,1)=N^{4}-8 N^{2}+6,
\end{gathered}
$$

Первые две строчки из этого списка воспроизводят выражения (4) и (5).

2.5. Аномалия 'т Хоофта-де Вита. Результаты, изложенные в данном пункте, можно найти в работах [45], [33].

Замечательным свойством формулы (13) является наличие полюсов при целых значениях размера матрицы $N$. Следует отметить, что их появление - это свойство фазы характеров. Ничего подобного в фазе Концевича в модели БГВ не происходит: как всегда, правильно определенная статистическая сумма Концевича фактически не зависит от $N$ [21], [33]. Как мы видели в п. 2.3, эти полюсы связаны с произволом в решении условий Вирасоро в обычной унитарной модели.

Фактически полюсы 'т Хоофта-де Вита в корреляторах отсутствуют: одновременно со знаменателями обращаются в нуль и числители, так что сингулярности разрешаются по правилу Лопиталя. Это, однако, не означает, что в вычислениях не требуется осторожности. Например, в (5) можно рассмотреть

$$
\int U_{11}^{2}\left(U_{11}^{\dagger}\right)^{2}[d U]=\frac{2\left(N^{2}-N\right)}{N^{2}\left(N^{2}-1\right)}=\frac{2}{N(N+1)} \stackrel{[N=1]}{=} 1,
$$

что полностью согласуется с (6). Однако, если сначала взять след в (5) и положить $N=1$ уже после этого, то (6) не воспроизведется:

$$
\int \operatorname{tr} U^{2} \operatorname{tr}\left(U^{\dagger}\right)^{2}[d U] \stackrel{(5)}{=} \frac{2\left(N^{2} \cdot N^{2}-N \cdot N\right)}{N^{2}\left(N^{2}-1\right)}=2 \neq 2-\delta_{N 1} .
$$

Аномалии подобного рода хорошо знакомы из применений размерной регуляризации, где вычисление следов и стремление размерности к критической часто оказываются некоммутирующими операциями.

2.6. Модель БГВ в контексте М-теории матричных моделей. Как объясняется в работах [16] (см. также более ранние результаты в [46] и [9]), статистическая сумма любой матричной модели в любой из ее фаз может быть разложена на элементарные составляющие, каждая из которых принадлежит семейству ОМК [21]. Наиболее важным строительным блоком в такой конструкции является исходная кубическая тау-функция Концевича $\tau_{\mathrm{K}}$, однако тау-функция $\tau_{\mathrm{BGW}}$, построенная из $Z_{\mathrm{BGW}}$ в фазе Концевича, оказывается второй по значимости (например, она возникает вместе с $\tau_{\mathrm{K}}$ при разложении на элементарные составляющие комплексной матричной модели). И $\tau_{\mathrm{K}}$, и $\tau_{\mathrm{BGW}}$ удовлетворяют одним и тем же непрерывным 
условиям Вирасоро [23] с небольшой разницей: $n \geqslant-1$ в случае $\tau_{\mathrm{K}}$ и $n \geqslant 0$ в случае $\tau_{\mathrm{BGW}}$. Второе, компенсирующее, отличие состоит в том, что вместо сдвига второй временно́й переменной в функции $\tau_{\mathrm{K}}$ в функции $\tau_{\mathrm{BGW}}$ сдвигается первая, в результате уменышенный набор условий по-прежнему однозначно определяет статистическую сумму. Подробности этой важной конструкции изложены в недавней работе [42].

2.7. Другие корреляторы и представления. Модель БГВ можно слегка обобщить, сделав в (10) степень в $\operatorname{det} S^{N}$ дополнительным свободным параметром. На этом пути мы получаем знаменитый интеграл Лейтвилера-Смилги [47]

$$
Z_{\mathrm{BGW}}(\nu ; t)=\int(\operatorname{det} U)^{\nu} e^{\operatorname{tr}\left(J^{\dagger} U+J U^{\dagger}\right)}[d U],
$$

который часто встречается в приложениях и широко рассматривается в литературе.

Другую модификацию можно получить, изменив представление. В формуле (10) статистическая сумма определена как генератор интегралов по унитарным матрицам в фундаментальном представлении размерности $N$ группы $G L(N)$, но вместо этого можно рассмотреть произвольное представление $R$ размерности $D_{R}(N)$. Все корреляторы, конечно, будут связаны с корреляторами в фундаментальном представлении, но связь будет не вполне тривиальной, и значения корреляторов будут совсем другими. Например, в произвольном представлении вместо (4) мы получим

$$
\int \mathcal{U}_{a b} \mathcal{U}_{c d}^{\dagger}[d U]=\frac{\delta_{a d} \delta_{b c}}{D_{R}}
$$

Более того, различные представления ортогональны в том смысле, что

$$
\int \mathcal{U}_{R} \mathcal{U}_{R^{\prime}}^{\dagger}[d U] \sim \delta_{R R^{\prime}}
$$

Важным методом работы с такого рода обобщениями является развитая теория разложений по характерам. Истоки этой теории можно найти в фундаментальных трудах Фробениуса, а сама она широко применяется в современных исследованиях, от чистой математики до прикладной физики. Что касается ссылок, то лучшее, что можно сделать, - это указать некоторые основные учебники [48] и лишь несколько связанных с этой темой работ в литературе по матричным моделям [2], [3]. Фактическое изложение этого предмета в следующем разделе в основном следует статье [40], а конкретные приложения теории характеров к модели БГВ и модели ИЗ в разделе 4 будут обсуждаться на материале работ [32], [33] и [49].

\section{3. ОСНОВЫ ИСЧИСЛЕНИЯ ХАРАКТЕРОВ}

3.1. Три определения характеров. Характер зависит от представления $R$ и от класса сопряженности группового элемента; он может быть представлен как функция либо "временнь́х" переменных $t_{1}, t_{2}, \ldots$, либо через вспомогательную матричную переменную Мивы $X$. В соответствии с этим мы будем пользоваться двумя обозначениями $\chi_{R}(t)=\chi_{R}[X]$. 
Представления $R$ группы $G L(\infty)$ нумеруются диаграммами Юнга, т.е. упорядоченными целочисленными разбиениями $R: \lambda_{1} \geqslant \lambda_{2} \geqslant \cdots \geqslant 0$, где все $\lambda_{j}$ - неотрицательные целые числа, $|R|$ - число клеток в диаграмме, $\sum_{j} \lambda_{j}=|R|$.

Временны́е переменные образуют полубесконечную последовательность, которую можно при желании рассматривать как последовательность собственных значений полубесконечной матрицы из $G L(\infty)$. Переменные Мивы задают $N$-мерное подпространство в пространстве временны́х переменных

$$
t_{k}=\frac{1}{k} \operatorname{tr} X^{k}=\frac{1}{k} \sum_{i} x_{i}^{k}
$$

параметризованное собственными значениями $x_{i}$ матрицы $X$ размера $N \times N$. Здесь и далее $\operatorname{tr}$ без дополнительных индексов обозначает след матрицы размера $N \times N$, т.е. след в первом фундаментальном представлении группы $G L(N)$, ассоциированном с одноклеточной диаграммой Юнга $R=[1]$. Та же матрица $X$ может быть записана в произвольном представлении $R$ группы $S L(N)$, мы будем обозначать этот ее образ $\mathcal{X}^{(R)}$. Хотя $X$ можно считать не зависящей от $N$, т.е. определенной как элемент группы $G L(\infty)$ так, чтобы $N$ было просто числом ненулевых собственных значений, ее образ $\mathcal{X}^{(R)}$ зависит от $N$ довольно сильно, поэтому следует проявлять осторожность с формулами, включающими $\mathcal{X}^{(R)}$. Однако во многих случаях вся зависимость от $N$ входит только через размерность $D_{R}$ представления $R$ группы $S L(N)$ (см. формулу (24) ниже в тексте). Иными словами, хотя и $\mathcal{X}^{(R)}$, и $D_{R}$ существенно зависят от $N$, если переписать формулы в терминах этих величин, то $N$ отдельно уже не появляется. С учетом этого обстоятельства мы опускаем индекс $N$ в наших обозначениях для $\mathcal{X}^{(R)}$ и $D_{R}$.

Имеются три важных определения характеров:

- первая детерминантная формула Вейля

$$
\chi_{R}(t)=\operatorname{det}_{i j}\left(s_{\lambda_{i}-i+j}(t)\right)
$$

выражающая $\chi_{R}(t)$ через детерминант полиномов Шура, которые определены равенством

$$
\exp \left(\sum_{k} t_{k} z^{k}\right)=\sum_{k} s_{k}(t) z^{k}
$$

• вторая детерминантная формула Вейля

$$
\chi_{R}(t)=\chi_{R}[X]=\frac{\operatorname{det}_{i j} x_{i}^{\lambda_{j}+N-j}}{\Delta(X)}=\frac{\operatorname{det}_{i j} x_{i}^{\lambda_{j}-j}}{\operatorname{det}_{i j} x_{i}^{-j}},
$$

где $\Delta(X)=\prod_{i<j}\left(x_{i}-x_{j}\right)=\operatorname{det}_{i j} x_{i}^{N-j}=\operatorname{det}_{i j} x_{i}^{j-1}-$ определитель Вандермонда; 
- формула следов

$$
\chi_{R}(t)=\chi_{R}[X]=\operatorname{Tr}_{R} \mathcal{X}^{(R)} .
$$

На самом деле подобно Янусу характеры имеют два лица. Они являются характерами двух существенно различных универсальных групп $G L(\infty)$ и $S(\infty)$ (через $S_{N}$ обозначена группа перестановок $N$ элементов). Тесная связь между двумя группами отражена уже в определении переменных Мивы, выражающем времена как симметрические функции собственных значений матрицы $X$.

Важную роль в исчислении характеров играют две дополнительные функции размерности представлений $S L(N)$ и $S_{N}$, связанных с данной диаграммой Юнга $R$ :

$$
\begin{array}{lll}
D_{R}=\operatorname{dim}_{R}(G L(N))=\chi_{R}\left(t_{k}\right)=\chi_{R}[I], & t_{k}=\frac{N}{k}, & N=|R|, \\
d_{R}=\frac{1}{N !} \operatorname{dim}_{R}(S(N))=\chi_{R}\left(t_{k}\right), & t_{k}=\delta_{k 1}, & N=|R| .
\end{array}
$$

Величина $d_{R}$ дается формулой крюков

$$
d_{R}=\prod \frac{1}{\text { hook length }}=\operatorname{det}_{1 \leqslant i, j \leqslant|R|} \frac{1}{\left(\lambda_{j}+i-j\right) !},
$$

где произведение вычисляется по всем клеткам диаграммы Юнга $R$, причем

$$
\frac{D_{R}}{d_{R}}=\prod_{i=1}^{N} \frac{\left(\lambda_{i}+N-i\right) !}{(N-i) !}
$$

Заметим, что величина $d_{R}$, полученная делением размерности на $N !=|R|$ !, зависит только от параметров $\lambda_{i}$, в то время как сама размерность $D_{R}$ содержит дополнительную явную зависимость от $N$.

Детерминант матрицы, т.е. произведение всех ее собственных значений, тоже равен характеру, а именно характеру абсолютно антисимметрического представления $R=[\underbrace{1, \ldots, 1}_{N}]:$

$$
\operatorname{det} X=\chi_{1 \ldots 1}[X], \quad \operatorname{rank} X=N \text {. }
$$

Более того, все целые степени детерминанта - это тоже характеры:

$$
(\operatorname{det} X)^{\nu}=\chi_{\nu \ldots \nu}[X]
$$

Если $\operatorname{rank} X=N$, то выраженные через $X$ времена $t_{k}$ с $k>N$ являются алгебраическими функциями времен с $k \leqslant N$. Соотношение задается обычным итерационным правилом

$$
\oint \operatorname{det}(1-s X) \frac{d s}{s^{k+1}}=\oint \exp \left(-\sum_{m \geqslant 0} s^{m} t_{m}\right) \frac{d s}{s^{k+1}}=0, \quad k>N .
$$


Будучи ограниченными на это подпространство, характеры с $|R|>N$ также становятся алгебраическими функциями характеров с $|R| \leqslant N$.

3.2. Правила сумм и соотношения ортогональности. Характеры удовлетворяют различным "правилам сумм": некоторые суммы по всем представлениям (т.е. по всем диаграммам Юнга или упорядоченным целочисленным разбиениям) равны каким-то иным известным величинам. K настоящему времени в приложениях возникают и широко используются следующие три правила сумм:

$$
\begin{aligned}
\sum_{R} d_{R} \chi_{R}(t) & =e^{t_{1}}=e^{\operatorname{tr} X} \\
\sum_{R} \chi_{R}(t) \chi_{R}\left(t^{\prime}\right) & =\exp \left(\sum_{k=1}^{\infty} k t_{k} t_{k}^{\prime}\right)=\frac{1}{\operatorname{Det}\left(I \otimes I-X \otimes X^{\prime}\right)}, \\
\sum_{R} \frac{d_{R}}{D_{R}} \chi_{R}[X] \chi_{R}\left[X^{\prime}\right] & =\int e^{\operatorname{tr} X U X^{\prime} U^{\dagger}}[d U] .
\end{aligned}
$$

Первая сумма есть частный случай второй, поскольку $d_{R}=\chi_{R}\left(\delta_{k 1}\right)$. Третья сумма (30) фактически является разложением по характерам интеграла ИЗ, но она может эффективно использоваться и в качестве правила сумм.

Очень важную роль в формализме теории характеров играет условие ортогональности: если $|R|=\left|R^{\prime}\right|$, то

$$
\chi_{R}(\tilde{\partial}) \chi_{R^{\prime}}(t)=\delta_{R, R^{\prime}} .
$$

Здесь в аргумент первого характера $\chi_{R}(t)$ (полинома от своих аргументов) вместо $t_{k}$ подставлены производные по временам $\tilde{\partial}_{k} \equiv \partial / \partial p_{k}=k^{-1} \partial / \partial t_{k}$. Например (см. п. 3.3),

$$
\begin{array}{lll}
\chi_{1}(t)=t_{1} & \Longrightarrow & \chi_{1}(\tilde{\partial})=\frac{\partial}{\partial t_{1}}, \\
\chi_{2}(t)=t_{2}+\frac{t_{1}^{2}}{2} & \Longrightarrow & \chi_{2}(\tilde{\partial})=\frac{1}{2}\left(\frac{\partial}{\partial t_{2}}+\frac{\partial^{2}}{\partial t_{1}^{2}}\right), \\
\chi_{11}(t)=-t_{2}+\frac{t_{1}^{2}}{2} & \Longrightarrow & \chi_{11}(\tilde{\partial})=\frac{1}{2}\left(-\frac{\partial}{\partial t_{2}}+\frac{\partial^{2}}{\partial t_{1}^{2}}\right),
\end{array}
$$

и нетрудно проверить, как условие ортогональности (31) работает в этих простейших случаях с $|R|=1,2$. На этих же примерах легко убедиться, что при $|R| \neq\left|R^{\prime}\right|$ соотношение ортогональности неверно.

3.3. Примеры характеров. Для иллюстрации мы приведем здесь в явном виде несколько первых характеров вместе с другими существенными характеристиками соответствующих представлений $R$ двух групп $G L$ и $S$. 


\begin{tabular}{|c|c|c|c|c|c|c|c|c|c|c|c|c|}
\hline 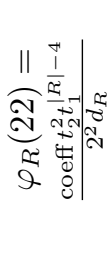 & 0 & 0 & 00 & 0 & 0 & 0 & $\infty$ & I & . & I & $\infty$ & 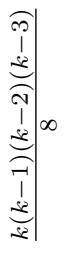 \\
\hline 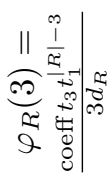 & 0 & 0 & 00 & 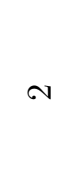 & I & $N$ & $\infty$ & 0 & $\stackrel{+}{i}$ & 0 & $\infty$ & 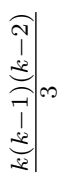 \\
\hline 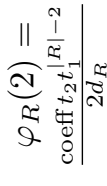 & 0 & 0 & $\neg$ I & $\infty$ & 0 & $?$ & 0 & $N$ & 0 & $\stackrel{\sim}{1}$ & i & $\begin{array}{c}3 \\
1 \\
3 \\
3 \\
2\end{array} \mid$ N \\
\hline 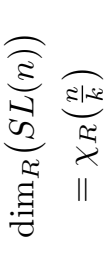 & $\neg$ & $\varepsilon$ & 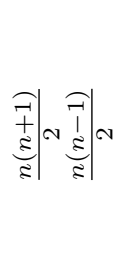 & 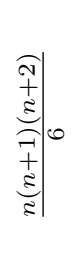 & 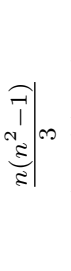 & 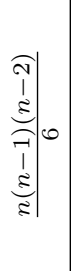 & 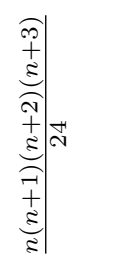 & 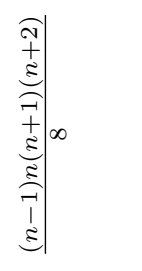 & 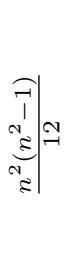 & 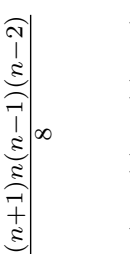 & 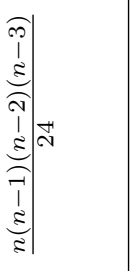 & 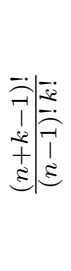 \\
\hline 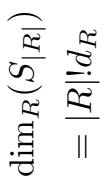 & $\neg$ & $\neg$ & $\neg-$ & -1 & $N$ & -1 & -1 & $\infty$ & $\sim$ & $\infty$ & -1 & -1 \\
\hline $\int_{0}^{\frac{7}{x}}$ & $\neg$ & -1 & $-|N-| N \mid$ & -10 & -100 & -10 & $-\mid \vec{N}$ & $-1 \infty$ & $-\mid \mp$ & $-1 \infty$ & -||$_{N}$ & $-\mid \equiv$ \\
\hline$\stackrel{2}{x}$ & $\neg$ & $\infty$ & 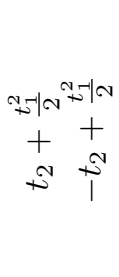 & 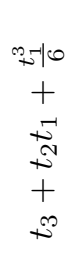 & $\begin{array}{c}0 \\
m-1 \infty \\
+\infty \\
+\infty\end{array}$ & 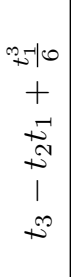 & 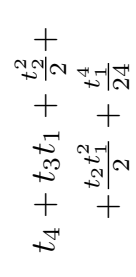 & 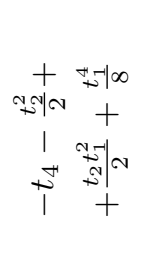 & 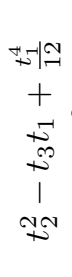 & 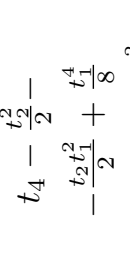 & 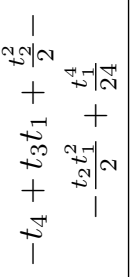 & \\
\hline$\approx$ & e & $\overparen{\beth}$ & 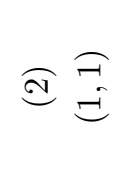 & $\overparen{(2}$ & ન્ & $\begin{array}{l}\overparen{I} \\
\stackrel{-}{\rightleftarrows}\end{array}$ & ક્ & $\widehat{\overparen{7}}$ & 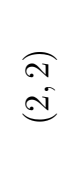 & $\stackrel{\text { İ }}{\stackrel{i}{I}}$ & 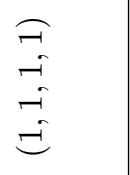 & 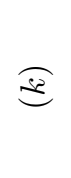 \\
\hline
\end{tabular}


3.4. Пример: уравнение $(20)$ для $G L(2)$. Построение матрицы $\mathcal{X}^{(R)}$ в произвольном представлении по матрице $X$, заданной в фундаментальном представлении, является довольно хитрым упражнением. На самом деле в явной форме уравнение $(20)$ - это соотношение между унитарными матрицами: $\chi_{R}\left[e^{A}\right]=\operatorname{Tr}_{R} e^{\mathcal{A}^{(R)}}$. Соотношение между $A$ и $\mathcal{A}^{(R)}$ устроено просто: это матрицы размеров $n \times n$ и $D_{R} \times D_{R}$ соответственно, выраженные через один и тот же набор параметров $u_{a}$ :

$$
A=i \sum_{a=0}^{n^{2}-1} u_{a} T_{a}^{(1)}, \quad \mathcal{A}^{(R)}=i \sum_{a=0}^{n^{2}-1} u_{a} T_{a}^{(R)} .
$$

Здесь $T_{a}^{(R)}$ - это генераторы алгебры $G L(n)$ в представлении $R$, а $R=[1]$ отвечает первому фундаментальному представлению.

Например, в случае $G L(2)$

$$
A=\left(\begin{array}{cc}
a+d & b \\
c & a-d
\end{array}\right),
$$

и мы имеем

$$
\begin{aligned}
& \chi_{1}\left[e^{A}\right]=t_{1}=\operatorname{Tr}_{D_{1}} e^{A}=\operatorname{Tr}_{2}\left\{\exp \left(\begin{array}{cc}
a+d & b \\
c & a-d
\end{array}\right)\right\}, \quad D_{1}=2, \\
& \chi_{2}\left[e^{A}\right]=t_{2}+\frac{t_{1}^{2}}{2}=\frac{1}{2} \operatorname{Tr}_{2} e^{2 A}+\frac{1}{2}\left(\operatorname{Tr}_{2} e^{A}\right)^{2}= \\
& =\operatorname{Tr}_{D_{2}}\left\{\exp \left(\begin{array}{ccc}
2 a+2 d & b & 0 \\
2 c & 2 a & 2 b \\
0 & c & 2 a-2 d
\end{array}\right)\right\}, \quad D_{2}=3, \\
& \chi_{11}\left[e^{A}\right]=-t_{2}+\frac{t_{1}^{2}}{2}=-\frac{1}{2} \operatorname{Tr}_{2} e^{2 A}+\frac{1}{2}\left(\operatorname{Tr}_{2} e^{A}\right)^{2}=e^{2 a}, \quad D_{11}=1, \\
& \chi_{3}\left[e^{A}\right]=t_{3}+t_{2} t_{1}+\frac{t_{1}^{3}}{6}=\frac{1}{3} \operatorname{Tr}_{2} e^{3 A}+\frac{1}{2}\left(\operatorname{Tr}_{2} e^{2 A}\right)\left(\operatorname{Tr}_{2} e^{A}\right)+\frac{1}{6}\left(\operatorname{Tr}_{2} e^{A}\right)^{3}= \\
& =\operatorname{Tr}_{D_{3}}\left\{\exp \left(\begin{array}{cccc}
3 a+3 d & b & 0 & 0 \\
3 c & 3 a+d & 2 b & 0 \\
0 & 2 c & 3 a-d & 3 b \\
0 & 0 & c & 3 a-3 d
\end{array}\right)\right\}, \quad D_{3}=4, \\
& \chi_{21}\left[e^{A}\right]=-t_{3}+\frac{t_{1}^{3}}{3}=-\frac{1}{3} \operatorname{Tr}_{2} e^{3 A}+\frac{1}{3}\left(\operatorname{Tr}_{2} e^{A}\right)^{3}= \\
& =\operatorname{Tr}_{D_{21}}\left\{\exp \left(\begin{array}{cc}
3 a+d & b \\
c & 3 a-d
\end{array}\right)\right\}, \quad D_{21}=2, \\
& \chi_{111}\left[e^{A}\right]=t_{3}-t_{2} t_{1}+\frac{t_{1}^{3}}{6}= \\
& =\frac{1}{3} \operatorname{Tr}_{2} e^{3 A}-\frac{1}{2}\left(\operatorname{Tr}_{2} e^{2 A}\right)\left(\operatorname{Tr}_{2} e^{A}\right)+\frac{1}{6}\left(\operatorname{Tr}_{2} e^{A}\right)^{3}=0, \quad D_{111}=0,
\end{aligned}
$$


Здесь следует обратить внимание на следующие обстоятельства:

- матрица $A$ совершенно не обязательно должна быть антиэрмитовой, приведенные выше соотношения верны для произвольной $A$;

- нормировка центрального (принадлежащего центру алгебры) $U(1)$-генератора включает в себя зависящий от представления множитель $|R|$;

- в общем случае для представления $G L(2)$ матрицами размера $r \times r$ можно использовать генераторы

$$
\begin{aligned}
T_{+}=\left(\begin{array}{ccccccc}
0 & 1 & 0 & \ldots & 0 & 0 \\
0 & 0 & 2 & \ldots & 0 & 0 \\
\vdots & \vdots & \vdots & \ddots & \vdots & \vdots \\
0 & 0 & 0 & \ldots & r-2 & 0 \\
0 & 0 & 0 & \ldots & 0 & r-1 \\
0 & 0 & 0 & \ldots & 0 & 0
\end{array}\right), \quad T_{-}=\left(\begin{array}{ccccccc}
0 & 0 & \ldots & 0 & 0 & 0 \\
r-1 & 0 & \ldots & 0 & 0 & 0 \\
0 & r-2 & \ldots & 0 & 0 & 0 \\
\vdots & \vdots & \ddots & \vdots & \vdots & \vdots \\
0 & 0 & \ldots & 2 & 0 & 0 \\
0 & 0 & \ldots & 0 & 1 & 0
\end{array}\right), \\
T_{3}=\left(\begin{array}{ccccc}
r-1 & 0 & \ldots & 0 & 0 \\
0 & r-3 & \ldots & 0 & 0 \\
\vdots & \vdots & \ddots & \vdots & \vdots \\
0 & 0 & \ldots & 3-r & 0 \\
0 & 0 & \ldots & 0 & 1-r
\end{array}\right), T_{0}=|R|\left(\begin{array}{ccccc}
1 & 0 & \ldots & 0 & 0 \\
0 & 1 & \ldots & 0 & 0 \\
\vdots & \vdots & \ddots & \vdots & \vdots \\
0 & 0 & \ldots & 1 & 0 \\
0 & 0 & \ldots & 0 & 1
\end{array}\right)
\end{aligned}
$$

с коммутационными соотношениями $\left[T_{+}, T_{-}\right]=T_{3},\left[T_{3}, T_{ \pm}\right]= \pm 2 T_{ \pm}$.

3.5. Характеры и симметрические функции. Здесь мы опираемся на результаты работ [32], [49].

Многие приложения характеров основаны на том, что в параметризации Мивы они становятся симметрическими функциями (от собственных значений $x_{i}$ матрицы $X)$ и при этом сами являются собственными функциями и собственными значениями некоторых выделенных операторов.

В пространстве симметрических функций имеется три "очевидных" набора однородных генераторов: времена $p_{k}[X]$, полиномы Шура $s_{k}[X]$ и элементарные симметрические полиномы $\sigma_{k}[X]$,

$$
\begin{aligned}
p_{k}[X] & =\sum_{i} x_{i}^{k}=\operatorname{tr} X^{k}, & k \geqslant 0, & p_{k}[X]=k t_{k}[X], \\
s_{k}[X] & =\sum_{i_{1} \leqslant \cdots \leqslant i_{k}} x_{i_{1}} \ldots x_{i_{k}}, & k \geqslant 1, & s_{0}[X]=1, \\
\sigma_{k}[X] & =\sum_{i_{1}<\cdots<i_{k}} x_{i_{1}} \ldots x_{i_{k}}, & k \geqslant 1, & \sigma_{0}[X]=1 .
\end{aligned}
$$

Этим трем базисам отвечают три производящие функции

$$
P(z)=\sum_{k=0}^{\infty} p_{k}[X] z^{k}=\operatorname{tr} \frac{I}{I-z X}
$$




$$
\begin{aligned}
& S(z)=\sum_{k=0}^{\infty} s_{k}[X] z^{k}=\prod_{i}\left(1-z x_{i}\right)^{-1}=\frac{1}{\operatorname{det}(I-z X)}, \\
& \Sigma(z)=\sum_{k=0}^{\infty} \sigma_{k}[X] z^{k}=\prod_{i}\left(1+z x_{i}\right)=\operatorname{det}(I+z X) .
\end{aligned}
$$

Они связаны друг с другом следующим образом:

$$
S(z)=\exp \left(\sum_{k=1}^{\infty} t_{k}[X] z^{k}\right)=\frac{1}{\operatorname{det}(I-z X)},
$$

где в силу $p_{k}[X]=k t_{k}[X]$

$$
P(z)=\operatorname{tr} I+z \frac{\partial}{\partial z} \ln E(z), \quad \Sigma(z)=\frac{1}{S(-z)} .
$$

Наборы $\left\{t_{k}\right\},\left\{s_{k}\right\}$ и $\left\{\sigma_{k}\right\}$ доставляют генераторы симметрических функций: любая такая функция является полилинейной комбинацией генераторов из любого набора. Линейные базисы образованы всевозможными произведениями генераторов и нумеруются диаграммами Юнга $R$. Очевидный выбор линейного базиса, составленного из мультипликативных генераторов, выглядит следующим образом:

$$
t^{R} \equiv \prod_{j} t_{\lambda_{j}} \equiv \prod_{j} t_{j}^{m_{j}} \quad \text { или } \quad p^{R} \equiv \prod_{j} p_{\lambda_{j}} \equiv \prod_{j} p_{j}^{m_{j}}=\left(\prod_{j} j^{m_{j}}\right) t^{R}=\mathrm{const} \cdot t^{R},
$$

$t_{\varnothing}=p_{\varnothing}=1$. Таким образом можно определить " $R$-степень" любой полубесконечной последовательности $\left\{p_{i}\right\}$, не обязательно образованной временны́ми переменными. Иногда полезен тот же базис, но с измененной нормировкой элементов:

$$
\widetilde{p^{R}} \equiv \frac{p^{R}}{z_{R}}, \quad z_{R} \equiv \prod_{j}\left(m_{j}\right) ! j^{m_{j}} .
$$

Заметим, что широкая тильда в этой формуле не вполне тождественна тильде в соотношениях ортогональности (31).

Менее тривиальный линейный базис образован характерами, построенными из генераторов $\left\{s_{k}\right\}$ по правилу (18), $\chi_{R}=\operatorname{det}_{i j}\left(s_{\lambda_{j}-j+i}\right)$. Оправданием для такого выбора базиса является следующее правило разложения [49]:

$$
\prod_{i}\left(\sum_{k=0}^{\infty} A_{k} x_{i}^{k}\right)=\sum_{R} \chi_{R}[X] \cdot \operatorname{det}_{i j}\left(A_{\lambda_{j}-j+i}\right) .
$$

Посредством второй формулы Вейля (19) оно связано с базовым детерминантным разложением [32], [49]

$$
\begin{gathered}
\operatorname{det}_{i j}\left(\sum_{k} A_{i k} B_{j k}\right)=\sum_{P \in S_{N}}(-1)^{P} \prod_{i=1}^{N} \sum_{k} A_{i k} B_{P(i) k}=\sum_{k_{1}, \ldots, k_{N}} \sum_{P}(-1)^{P} \prod_{i=1}^{N} A_{i k_{i}} B_{P(i) k_{i}}= \\
=\sum_{k_{1}>\cdots>k_{N}}\left(\sum_{P, P^{\prime}}(-1)^{P}(-1)^{P^{\prime}} \prod_{i=1}^{N} A_{P^{\prime}(i) k_{i}} B_{P(i) k_{i}}\right)=\sum_{k_{1}>\cdots>k_{N}} \operatorname{det}_{i j} A_{i k_{j}} \operatorname{det}_{i j} B_{i k_{j}},
\end{gathered}
$$


обобщающим равенство $\operatorname{det}(A B)=\operatorname{det} A \cdot \operatorname{det} B$ для квадратных матриц на случай прямоугольных. В самом деле, для $k_{j}=\lambda_{j}-j$ (заметим, что из $\lambda_{1} \geqslant \cdots \geqslant \lambda_{N}$ следует, что $\left.k_{1}=\lambda_{1}-1>\cdots>k_{N}=\lambda_{N}-N\right)$ и при $\chi_{R}[X]$, заданным формулой $(19)$, правая часть (36) в точности совпадает с правой частью (37). Поэтому

$$
\begin{aligned}
& \sum_{R} \chi_{R}[X] \cdot \operatorname{det}_{i j}\left(A_{\lambda_{j}-j+i}\right) \stackrel{(19)}{=} \sum_{\lambda_{1} \geqslant \cdots \geqslant \lambda_{N}} \frac{\operatorname{det}_{i j}\left(x_{i}^{\lambda_{j}-j}\right) \operatorname{det}_{i j}\left(A_{\lambda_{j}-j+i}\right)}{\operatorname{det}_{i j} x_{j}^{-i}} \stackrel{(37)}{=} \\
& \stackrel{(37)}{=} \frac{\operatorname{det}_{i j}\left(\sum_{k} A_{k+i} x_{j}^{k}\right)}{\operatorname{det}_{i j} x_{j}^{-i}}=\frac{\operatorname{det}_{i j}\left(A\left(x_{j}\right) x_{j}^{-i}\right)}{\operatorname{det}_{i j} x_{j}^{-i}}=\prod_{j} A\left(x_{j}\right),
\end{aligned}
$$

где $A(x)=\sum_{k=0}^{\infty} A_{k} x^{k}$, что завершает вывод (36) из (37). Отметим также, что пределы суммирования автоматически подбираются нужным образом.

3.6. Характеры как собственные функции и собственные значения. В этом пункте мы используем результаты работ [36], [38].

Характеры в параметризации Мивы $\chi_{R}[X]$ являются общими собственными функциями бесконечного набора операторов $\widehat{W}(R)$ [38]. Эти операторы находятся во взаимно однозначном соответствии с диаграммами Юнга (упорядоченными целочисленными разбиениями) и строятся из $G L$-генератора $\widehat{D}_{i j}=X_{i k} \partial / \partial X_{j k}$ в два шага: сначала определяется полубесконечная последовательность коммутирующих операторов $\widehat{D}_{k}=: \operatorname{tr} \widehat{D}^{k}$ : и затем по общему правилу (35) строятся операторы

$$
\widehat{W}(R)=: \widetilde{D^{R}}:=: \prod_{k} \frac{1}{m_{k} ! k^{m_{k}}}\left(\operatorname{tr} \widehat{D}^{k}\right)^{m_{k}}:
$$

Дополнительным элементом здесь является нормальное упорядочение, подразумевающее, что все $X$ стоят слева от всех $\partial / \partial X$. Согласно работе [38] эти операторы образуют центр универсальной обертывающей алгебры, $\left[\widehat{W}(R), \widehat{W}\left(R^{\prime}\right)\right]=0$ для всех $R, R^{\prime}$, и характеры являются их собственными функциями: для любых $R, R^{\prime}$

$$
\widehat{W}(R) \chi_{R^{\prime}}[X]=\varphi_{R^{\prime}}(R) \chi_{R^{\prime}}[X]
$$

Соответствующие собственные значения $\varphi_{R}(\Delta)$ на самом деле тоже оказываются характерами симметрической группы $S$, их можно определить как коэффициенты разложения одного линейного базиса по другому - разложения $\chi_{R}$ по переменным $p$ :

$$
\chi_{R}(p)=\sum_{|\Delta|=|R|} d_{R} \varphi_{R}(\Delta) p^{\Delta} .
$$

Сумма здесь берется по диаграммам Юнга $\Delta$, имеющим тот же размер (число клеток), что и $R$. Для $|\Delta|>|R|$ собственные значения равны нулю, а для $|\Delta|<|R|$ они определяются по такому правилу:

$$
\varphi_{R}(\Delta, \underbrace{1, \ldots, 1}_{k})=\frac{(|R|-|\Delta|) !}{(|R|-|\Delta|-k) ! k !} \varphi_{R}(\Delta, \underbrace{1, \ldots, 1}_{|R|-|\Delta|}), \quad \text { если } \quad 1 \notin \Delta,
$$


т.е. в этой формуле через $\Delta$ обозначена часть диаграммы Юнга, вообще не содержащая единичных столбцов. В частности, для такой $\Delta$ мы получаем $\varphi_{R}(\Delta)=$ $\varphi_{R}(\Delta, \underbrace{1, \ldots, 1}_{|R|-|\Delta|})$. Иными словами, если $\Delta$ содержит в точности $k$ единичных столбцОВ, то

$$
\varphi_{R}(\Delta)=\frac{(|R|-|\Delta|+k) !}{(|R|-|\Delta|) ! k !} \varphi_{R}(\Delta, \underbrace{1, \ldots, 1}_{|R|-|\Delta|}), \quad \text { если } \quad 1^{k} \in \Delta .
$$

В приложениях оказывается важным также и обращение формулы (41)

$$
\widetilde{p^{\Delta}}=\sum_{|R|=|\Delta|} d_{R} \chi_{R}(p) \varphi_{R}(\Delta)
$$

Отметим, что в левой части здесь появляется $\widetilde{p^{\Delta}}$, а не $p^{\Delta}$, как в правой части (41). Отметим также, что обе эти формулы записаны в терминах переменных $p_{k}$, а не $t_{k}$, разница между ними в множителе: $p_{k}=k t_{k}$.

Операторы $\widehat{W}(R)$ и тем самым их собственные значения образуют интересную коммутативную ассоциативную алгебру [38], [40]:

$$
\widehat{W}\left(R_{1}\right) \widehat{W}\left(R_{2}\right)=\sum_{R_{3}} C_{R_{1} R_{2}}^{R_{3}} \widehat{W}\left(R_{3}\right) \Longrightarrow \varphi_{R}\left(R_{1}\right) \varphi_{R}\left(R_{2}\right)=\sum_{R_{3}} C_{R_{1} R_{2}}^{R_{3}} \varphi_{R}\left(R_{3}\right)
$$

для любых $R_{1,2,3}$.

\section{4. ТЕОРИЯ ИНТЕГРАЛА ИЦИКСОНА-ЗЮБЕРА}

Важное приложение теория характеров находит в теории интегралов Хариш-Чандры-Ициксона-Зюбера (ХЧИЗ) [43], [44]. Как упоминалось во введении, этот интеграл является простейшей версией (основным строительным блоком) модели Казакова-Мигдала [27]-[33] для решеточной глюодинамики и фактически для многих других матрично-модельных подходов к непертурбативной динамике калибровочных теорий с распространяющимися (частицеподобными) степенями свободы. Интеграл ИЗ также является нетривиальным примером группового интеграла, который удовлетворяет требованиям теоремы Дустермаата-Хекмана [50] и к которому приложимы методы, известные ныне под именем техники локализации (одним из ярких достижений в этой области является инстантонное исчисление Некрасова [51] в теории Зайберга-Виттена [52]).

С интегралами ХЧИЗ можно работать различными методами, начиная прямо с тождеств Уорда [44]. Мы опишем здесь подход, основанный на теории характеров [32], [49] и тесно связанной с ней техникой ОМК из работ [29]-[33]. Этот подход был недавно заново рассмотрен в работе [14] и применен к старой (но все еще не решенной) задаче о вычислении произвольных корреляторов с весом ХЧИЗ [30], [31]. 
4.1. Интеграл ИЗ и формула дуальности. Интеграл ИЗ (или ХЧИЗ) - это унитарный интеграл с нетривиальным весом специального вида:

$$
J_{\mathrm{IZ}}(X, Y)=\int e^{\operatorname{tr} X U Y U^{\dagger}}[d U] .
$$

Этот интеграл выражается через собственные значения двух матриц $X$ и $Y$ размера $N \times N$ с помощью знаменитой детерминантной формулы [44]

$$
J_{\mathrm{IZ}} \sim \frac{\operatorname{det}_{i j} e^{x_{i} y_{j}}}{\Delta(X) \Delta(Y)}=\frac{1}{\Delta(X) \Delta(Y)} \sum_{P \in S_{N}}(-1)^{P} e^{x_{i} y_{P(i)}},
$$

где $\Delta(X)$ и $\Delta(Y)$ - два определителя Вандермонда.

При стандартном выводе формулы (47) надо заметить, что $J_{\mathrm{IZ}}$ зависит только от собственных значений матриц $X$ и $Y$, и подставить функцию от собственных значений в качестве анзаца в очевидные тождества Уорда:

$$
\begin{aligned}
& \left\{\operatorname{tr}\left(\frac{\partial}{\partial X^{\mathrm{T}}}\right)^{k}-\operatorname{tr} Y^{k}\right\} J_{\mathrm{IZ}}(X, Y)=0, \\
& \left\{\operatorname{tr}\left(\frac{\partial}{\partial Y^{\mathrm{T}}}\right)^{k}-\operatorname{tr} X^{k}\right\} J_{\mathrm{IZ}}(X, Y)=0, \quad k \geqslant 0,
\end{aligned}
$$

что превращает задачу об интеграле в задачу об уравнениях типа Калоджеро или Данкля, поскольку, например,

$$
\operatorname{tr}\left(\frac{\partial}{\partial X^{\mathrm{T}}}\right)^{2}=\sum_{i} \frac{\partial^{2}}{\partial x_{i}^{2}}+\sum_{i \neq j} \frac{1}{x_{i}-x_{j}}\left(\frac{\partial}{\partial x_{i}}-\frac{\partial}{\partial x_{j}}\right),
$$

когда этот оператор действует на функцию от собственных значений. Можно проверить, что (47) в самом деле есть решение этих уравнений. Данный метод является довольно трудоемким, и ответ ни в коей мере не очевиден, по крайней мере для тех, кто не знаком с уравнениями Калоджеро и Данкля и связанной с ними теорией “зональных сферических функций” [53]. Взамен этого у метода есть содержательные обобщения, одно из последних [54] - к теории интегральных дискриминантов (однородных негауссовых интегралов), важной ветви нелинейной алгебры [55].

Прямой путь от (46) к результату (47) дает подход Дустермаата-Хекмана [50], где используется то, что интеграл, инвариантный относительно действия компактной группы и удовлетворяющий определенным соотношениям между классическим действием и квантовой мерой, т.е. подчиненный условиям, которые тривиально выполняются для теоретико-группового интеграла (46), в точности равен своему полному квазиклассическому приближению - сумме (с чередующимися знаками) по всем экстремумам классического действия,

$$
\int e^{-S(\phi)} d \phi \sim \sum_{\phi_{0}: \delta S\left(\phi_{0}\right)=0}\left(\operatorname{det} \frac{\partial^{2} S\left(\phi_{0}\right)}{\partial \phi^{2}}\right)^{-1 / 2} e^{-S\left(\phi_{0}\right)} .
$$


Как объясняется в разделе 3.2 обзора [5] (см. также [14]), уравнения движения $\left[X, U Y U^{\dagger}\right]=0$ для действия $\operatorname{tr} X U Y U^{\dagger}$ после унитарных преобразований, диагонализующих $X$ и $Y$, имеют в качестве решений произвольные матрицы перестановки $U=P$ (число решений возрастает, когда некоторые собственные значения $X$ и $Y$ совпадают, но это не сказывается на ответе). Тогда теорема Дустермаата-Хекмана (50) означает, что

$$
J_{\mathrm{IZ}}(X, Y) \sim \sum_{P} \frac{(-1)^{P}}{\Delta(X) \Delta(Y)} \exp \left(\sum_{i} x_{i} y_{P(i)}\right),
$$

где определители Вандермонда возникают из предэкспоненциального множителя, а знаковый множитель $(-1)^{P}$ естественно появляется при извлечении квадратного корня и различает вклады максимумов и минимумов в квазиклассическую сумму. Метод Дустермаата-Хекмана, известный также как техника локализации, был открыт при изучении интегралов типа ХЧИЗ и впоследствии нашел множество разнообразных приложений. Одним из перспективных должно стать приложение к 3-алгебрам и связанной с ними теории Баггера-Ламберта-Густафсона [56], где этот метод остается единственным прямым подходом к определению какой-либо замены матричных моделей, поскольку никакой прямой замены матрицам в случае 3-алгебр пока не найдено.

Определенное значение имеет также следующее представление интеграла (46) интегралом по эрмитовой матрице [29], [33], [14]. Непосредственно из определения (46) получаем

$$
\begin{aligned}
J_{\mathrm{IZ}} & \stackrel{(46)}{=} \int e^{\operatorname{tr} X U Y U^{\dagger}}[d U] \stackrel{(2)}{=} \int e^{\operatorname{tr} S\left(I-M M^{\dagger}\right)+\operatorname{tr} X M Y M^{\dagger}} d^{2} M d S= \\
& =\int \frac{e^{\operatorname{tr} S} d S}{\operatorname{det}_{N^{2} \times N^{2}}(S \otimes I-X \otimes Y)} .
\end{aligned}
$$

Легко перейти к базису, где, скажем, матрица $Y$ диагональна, $Y=\operatorname{diag}\left\{y_{k}\right\}$. Тогда

$$
\begin{aligned}
J_{\mathrm{IZ}}(X, Y) & \sim \int \frac{e^{\operatorname{tr} S} d S}{\prod_{k} \operatorname{det}\left(S-y_{k} X\right)} \longrightarrow \int \frac{e^{\operatorname{tr} S X} d S}{\prod_{k} \operatorname{det}\left(S-y_{k} I\right)} \sim \\
& \sim \int J_{\mathrm{IZ}}(X, S) \frac{\prod_{i \neq j}\left(s_{i}-s_{j}\right) \prod_{i} d s_{i}}{\prod_{i, k}\left(s_{i}-y_{k}\right)}
\end{aligned}
$$

где определители и следы берутся у матриц размера $N \times N$, а стрелка соответствует замене переменных интегрирования $S \rightarrow S X$ (которая подразумевает изменение контура интегрирования по $S$ ). На последнем шаге мы переходим к интегралу по собственным значениям $S$, а угловой интеграл снова дается тем же самым интегралом ИЗ. Согласно [14] это соотношение дуальности позволяет получить эффективную рекурсию по размеру $N$ унитарной матрицы. 
4.2. Разложение интеграла ХЧИЗ по характерам. Пользуясь исчислением характеров, мы можем немедленно вывести альтернативную формулу - разложение по характерам. Воспользовавшись (28), можно выразить $e^{\operatorname{tr} \Psi}$ с $\Psi \equiv X U Y U^{\dagger}$ как линейную комбинацию характеров, $e^{\operatorname{tr} \Psi}=\sum_{R} d_{R} \chi_{R}(\Psi)$, и после этого усреднить характеры по унитарной группе:

$$
\int \chi_{R}[\Psi][d U] \stackrel{(20)}{=} \int \operatorname{Tr}_{R} \Psi_{R}[d U] \stackrel{(20)}{=} \int \operatorname{Tr}_{R}\left(\mathcal{X}_{R} \mathcal{U} \mathcal{Y}_{R} \mathcal{U}^{\dagger}\right)[d U] \stackrel{(16)}{=} \frac{1}{D_{R}} \chi_{R}[X] \chi_{R}[Y]
$$

В результате

$$
J_{\mathrm{IZ}}=\int e^{\operatorname{tr} \Psi}[d U] \stackrel{(28)}{=} \sum_{R} d_{R} \int \chi_{R}[\Psi][d U] \stackrel{(54)}{=} \sum_{R} \frac{d_{R} \chi_{R}[X] \chi_{R}[Y]}{D_{R}} .
$$

Эта элементарная, но мощная формула приведена, например, в работе [49] и (с ошибочно пропущенным множителем $d_{R}$ ) в работе [3]. Недавнее ее приложение к точному вычислению корреляторов в гауссовой эрмитовой модели см. в последних статьях в ссылке [17]. Аналогичным образом можно получить разложение по характерам в более сложном случае, когда в экспоненте стоят два слагаемых. Этот случай известен как (обобщенный) интеграл Березина-Карпелевича [57], [49]

$$
\begin{aligned}
J_{\mathrm{BK}} & =\iint e^{X U Y V^{\dagger}+V Y U^{\dagger} X}[d U][d V]= \\
& =\sum_{R, R^{\prime}} d_{R} d_{R^{\prime}} \iint \chi_{R}\left(X U Y V^{\dagger}\right) \chi_{R^{\prime}}\left(V Y U^{\dagger} X\right)[d U][d V]= \\
& =\sum_{R} d_{R}^{2} \iint \operatorname{Tr}_{R}\left(\mathcal{X}_{R} \mathcal{U} \mathcal{Y}_{R} \mathcal{V}^{\dagger}\right) \operatorname{Tr}_{R}\left(\mathcal{X}_{R} \mathcal{V} \mathcal{Y}_{R} \mathcal{U}^{\dagger}\right)[d U][d V]= \\
& =\sum_{R} \frac{d_{R}^{2}}{D_{R}^{2}} \chi_{R}\left[X^{2}\right] \chi_{R}\left[Y^{2}\right],
\end{aligned}
$$

где последнее равенство имеет место в силу (16), предпоследнее - в силу (17) и на последнем шаге использовано вытекающее из (17) правило

$$
\int \chi_{R}[U] \chi_{R^{\prime}}[U][d U]=\delta_{R, R^{\prime}}
$$

О приложениях к двухматричной модели см., например, статью [3].

Представление (47) получается из (55), если выразить характеры в правой части равенства через собственные значения с помощью второй формулы Вейля (19):

$$
\begin{aligned}
J_{\mathrm{IZ}} \stackrel{(55)}{=} \sum_{R} \frac{d_{R} \chi_{R}[X] \chi_{R}[Y]}{D_{R}} \stackrel{(19)}{=} \frac{1}{\Delta(X) \Delta(Y)} \sum_{R} \frac{d_{R}}{D_{R}} \operatorname{det}_{i j} x_{i}^{N-j+\lambda_{j}} \operatorname{det}_{i j} y_{i}^{N-j+\lambda_{j}} \stackrel{(24)}{=} \\
\stackrel{(24)}{=} \frac{1}{\Delta(X) \Delta(Y)} \sum_{\lambda_{1} \geqslant \cdots \geqslant \lambda_{N} \geqslant 0} \prod_{i=1}^{N} \frac{(N-i) !}{\left(\lambda_{i}+N-i\right) !} \operatorname{det}_{i j} x_{i}^{N-j+\lambda_{j}} \operatorname{det}_{i j} y_{i}^{N-j+\lambda_{j}\left[\lambda_{i}+N-i=k_{i}\right]}= \\
\stackrel{\left[\lambda_{i}+N-i=k_{i}\right]}{\quad \frac{\prod_{i=1}^{N}(N-i) !}{\Delta(X) \Delta(Y)}} \sum_{k_{1}>\cdots>k_{N}} \frac{\operatorname{det}_{i j} x_{i}^{k_{j}} \operatorname{det}_{i j} y_{i}^{k_{j}}}{\prod_{j} k_{j} !}=\left(\prod_{j=0}^{N-1} j !\right) \frac{\operatorname{det}_{i j} e^{x_{i} y_{j}}}{\Delta(X) \Delta(Y)},
\end{aligned}
$$


потому что, как частный случай (37),

$$
\sum_{k_{1}>\cdots>k_{N}}\left(\prod_{j=1}^{N} a_{j}\right) \operatorname{det}_{i j} x_{i}^{k_{j}} \operatorname{det}_{i j} y_{i}^{k_{j}}=\frac{1}{N !} \sum_{P, P^{\prime}} \sum_{k_{j}}\left(\phi_{P(j)} \psi_{P^{\prime}(j)}\right)^{k_{j}}=\operatorname{det}_{i j} A\left(\phi_{i} \psi_{j}\right)
$$

для $A(x)=\sum_{k} a_{k} x^{k}[49]$.

Точно так же из (56) мы получаем еще одну знаменитую формулу [57], [49]:

$$
\begin{aligned}
J_{\mathrm{BK}} & \stackrel{(59)}{=} \sum_{R} \frac{d_{R}^{2} \chi_{R}\left[X^{2}\right] \chi_{R}\left[Y^{2}\right]}{D_{R}^{2}}=\frac{\left(\prod_{j=0}^{N-1} j !\right)^{2}}{\Delta\left(X^{2}\right) \Delta\left(Y^{2}\right)} \sum_{k_{1}>\cdots>k_{N}} \frac{\operatorname{det}_{i j} x_{i}^{2 k_{j}} \operatorname{det}_{i j} y_{i}^{2 k_{j}}}{\left(\prod_{j} k_{j} !\right)^{2}} \stackrel{(59)}{=} \\
& \stackrel{(59)}{=}\left(\prod_{j=0}^{N-1} j !\right)^{2} \frac{\operatorname{det}_{i j} \mathcal{I}_{0}\left(2 x_{i} y_{j}\right)}{\Delta\left(X^{2}\right) \Delta\left(Y^{2}\right)},
\end{aligned}
$$

где функция Бесселя $\mathcal{I}_{0}(2 x)=\sum_{k} \frac{x^{2 k}}{(k !)^{2}}$. Данное соотношение можно рассматривать как транцендентное обощение интеграла ИЗ. Дальнейшее обобщение найдено в работе [58]:

$$
\begin{aligned}
& \iint \operatorname{det}^{\nu}(U V) e^{A U B V^{\dagger}+V C U^{\dagger} D}[d U][d V]= \\
& =\left(\prod_{j=1}^{N-1} j !\right)^{2}\left(\frac{\operatorname{det}(C D)}{\operatorname{det}(A B)}\right)^{\nu / 2} \frac{\operatorname{det} \mathcal{I}_{\nu}\left(2 x_{i} y_{j}\right)}{\Delta\left(X^{2}\right) \Delta\left(Y^{2}\right)}
\end{aligned}
$$

где на этот раз $x_{i}^{2}$ и $y_{i}^{2}$ - это собственные значения произведений $A D$ и $C B$. Теперь можно даже разрешить матрицам $U$ и $V$ иметь разные размеры $N$ и $M$ [58]. Тогда матрицы $A, B, C, D$ окажутся прямоугольными, параметр $\nu$ будет отсутствовать и для $M \leqslant N$ получим

$$
\begin{aligned}
& \int_{N \times N} \int_{M \times M} e^{A U B V^{\dagger}+V C U^{\dagger} D}[d U][d V]= \\
& \quad=\left(\prod_{j=N-M}^{N-1} j ! \prod_{l=N-M}^{N-1} l !\right) \frac{\operatorname{det} \mathcal{I}_{N-M}\left(2 x_{i} y_{j}\right)}{\Delta\left(X^{2}\right) \Delta\left(Y^{2}\right) \prod_{i=1}^{M}\left(x_{i} y_{i}\right)^{N-M}},
\end{aligned}
$$

где $x_{i}^{2}, y_{i}^{2}$ - собственные значения меньших по размеру $(M \times M)$-матриц $A D$ и $C B$. Дальнейшие обобщения на суперматрицы описаны в работе [59].

4.3. Редукция к модели БГВ и интеграл Лейтвиллера-Смилги. Аналогичные разложения по характерам имеются, естественно, и для более простой модели БГВ. Однако, поскольку интеграл (10) содержит два слагаемых в показателе экспоненты, он на самом деле ближе к трансцендентному интегралу Березина-Карпелевича, нежели к интегралу ИЗ. Вместо (56) для (10) мы получим

$$
Z_{\mathrm{BGW}}=\int e^{\operatorname{tr}\left(J^{\dagger} U+J U^{\dagger}\right)}[d U]=\sum_{R, R^{\prime}} d_{R} d_{R^{\prime}} \int \chi_{R}\left[J^{\dagger} U\right] \chi_{R^{\prime}}\left[J U^{\dagger}\right][d U] \stackrel{(17)}{=} \sum_{R} \frac{d_{R}^{2}}{D_{R}} \chi_{R}\left[J J^{\dagger}\right] .
$$


Чтобы сделать следующий шаг [33], [49], потребуется не только (24), но и формула крюков (23):

$$
\begin{aligned}
Z_{\mathrm{BGW}} & =\sum_{R} \frac{d_{R}^{2}}{D_{R}} \chi_{R}\left[J J^{\dagger}\right]=\frac{\prod_{j=0}^{N-1} j !}{\Delta\left[J J^{\dagger}\right]} \sum_{k_{1}>\cdots>k_{N}} \frac{\operatorname{det}_{i j} J_{i}^{2 k_{j}}}{\left(\prod_{j} k_{j} !\right)} \operatorname{det}_{i j} \frac{1}{\left(k_{j}-N+i\right) !} \stackrel{(37)}{=} \\
& \stackrel{(37)}{=}\left(\prod_{j=0}^{N-1} j !\right) \frac{\operatorname{det}_{i j}\left(J_{j}^{N-i} \mathcal{I}_{i-N}\left(2 J_{j}\right)\right)}{\Delta\left(J J^{\dagger}\right)}=\left(\prod_{j=0}^{N-1} j !\right) \frac{\operatorname{det}_{i j}\left(J_{i}^{j-1} \mathcal{I}_{j-1}\left(2 J_{i}\right)\right)}{\Delta\left(J J^{\dagger}\right)},
\end{aligned}
$$

где $J_{i}^{2}$ - это собственные значения эрмитовой матрицы $J J^{\dagger}$, а функция Бесселя

$$
\mathcal{I}_{s}(2 x)=\sum_{k=0}^{\infty} \frac{x^{2 k+s}}{k !(k+s) !}=\mathcal{I}_{-s}(2 x)
$$

Интеграл Лейтвиллера-Смилги (15) можно преобразовать аналогичным образом, нужно только подходящее обобщение соотношений (28) и (23):

$$
(\operatorname{det} U)^{\nu} e^{\operatorname{tr} U}=\sum_{\lambda_{1} \geqslant \cdots \geqslant \lambda_{N} \geqslant 0} \chi_{\lambda_{1} \ldots \lambda_{N}}[U] \operatorname{det}_{i j} \frac{1}{\left(\lambda_{j}-\nu+i-j\right) !}
$$

Из этой формулы получаем [47], [49]

$$
\begin{aligned}
Z_{\mathrm{BGW}}(\nu ; t) & =\int(\operatorname{det} U)^{\nu} e^{\operatorname{tr}\left(J^{\dagger} U+J U^{\dagger}\right)}[d U]= \\
& =\frac{\prod_{j=0}^{N-1} j !}{\Delta\left[J J^{\dagger}\right]} \sum_{k_{1}>\cdots>k_{N}} \frac{\operatorname{det}_{i j} J_{i}^{2 k_{j}}}{\left(\prod_{j} k_{j} !\right)} \operatorname{det}_{i j} \frac{1}{\left(k_{j}-N-\nu+i\right) !}= \\
& =\left(\prod_{j=0}^{N-1} j !\right) \frac{\operatorname{det}_{i j}\left(J_{i}^{j-1} \mathcal{I}_{\nu+j-1}\left(2 J_{i}\right)\right)}{\Delta\left(J J^{\dagger}\right)} .
\end{aligned}
$$

4.4. Корреляторы матриц $\Psi$ в интеграле ИЗ. В данном пункте мы следуем результатам работы [40].

Разложение по характерам позволяет вычислить произвольные корреляторы следов матрицы $\Psi=X U Y U^{\dagger}$ и их степеней с действием ИЗ. Для диаграммы Юнга $\Delta=\left\{\delta_{1} \geqslant \delta_{2} \geqslant \cdots\right\}=\{\underbrace{m, \ldots, m}_{\alpha_{m}}, \ldots, \underbrace{1, \ldots, 1}_{\alpha_{1}}\}$ размера $|\Delta|=\sum_{k} \delta_{k}=\sum_{m} m \alpha_{m}$ мы получаем из (44), что

$$
\Psi^{\Delta} \equiv(\operatorname{tr} \Psi)^{\alpha_{1}}\left(\operatorname{tr} \Psi^{2}\right)^{\alpha_{2}} \ldots\left(\operatorname{tr} \Psi^{m}\right)^{\alpha_{m}}=z_{\Delta} \sum_{|R|=|\Delta|} d_{R} \chi_{R}(\Psi) \varphi_{R}(\Delta)
$$


Вместо (55) мы можем теперь написать

$$
\begin{aligned}
\int \Psi^{\Delta} e^{\operatorname{tr} \Psi}[d U] & =\sum_{n=0}^{\infty} \frac{1}{n !} \Psi^{\Delta+n \cdot 1}[d U] \stackrel{(67)}{=} \\
& \stackrel{(67)}{=} \sum_{n} \frac{z_{\Delta+n \cdot 1}}{n !} \sum_{|R|=|\Delta|+n} d_{R} \varphi_{R}(\Delta+n \cdot 1) \int \chi_{R}(\Psi)[d U] \stackrel{(54)}{=} \\
& \stackrel{(54)}{=} \sum_{R} \frac{z_{\Delta+(|R|-|\Delta|) \cdot 1}}{(|R|-|\Delta|) !} \frac{d_{R}}{D_{R}} \varphi_{R}(\Delta+(|R|-|\Delta|) \cdot 1) \chi_{R}[X] \chi_{R}[Y] .
\end{aligned}
$$

Отметим, что сумма по $n$ фактически заменена на сумму по всем $R$. При этом из суммы по всем $n$ отбирается только вклад с $n=|R|-|\Delta|$. Если в диаграмме Юнга $\Delta$ нет единичных столбцов, то $z_{\Delta+(|R|-|\Delta|) \cdot 1}=(|R|-|\Delta|) ! z_{\Delta}$, и сложная формула несколько упрощается:

$$
\int \widetilde{\Psi^{\Delta}} e^{\operatorname{tr} \Psi}[d U]=\sum_{R} \frac{d_{R}}{D_{R}} \varphi_{R}(\Delta+(|R|-|\Delta|) \cdot 1) \chi_{R}[X] \chi_{R}[Y], \quad \text { если } \quad 1 \notin \Delta .
$$

В качестве побочного продукта этого вычисления мы получаем доказательство соотношения (40) (см. работу [40]). Для этого подействуем оператором $\widehat{W}(\Delta)=$ $\widetilde{D}^{\Delta}$ на $J_{\text {IZ }}$. Оператор $\widehat{D}_{i j}=\sum_{k} X_{i k} \partial / \partial X^{j k}$ преобразует $\Psi_{k l}=\left(X U Y U^{\dagger}\right)_{k l}$ в себя, $\widehat{D}_{i j} \Psi_{k l}=\delta_{j k} \Psi_{i l}$, следовательно, мы имеем

$$
\widehat{W}(\Delta) J_{\mathrm{IZ}}(X, Y)=\widehat{W}(\Delta) \int e^{\operatorname{tr} \Psi}[d U]=\int \widetilde{\Psi^{\Delta}} e^{\operatorname{tr} \Psi}[d U],
$$

и теперь остается подставить соотношение (55) для $J_{\mathrm{IZ}}$ в левую часть равенства и формулу (68) для среднего - в правую. Приравнивая в обеих частях равенства коэффициенты перед $d_{R} \chi_{R}[Y] / D_{R}$, получаем

$$
\widehat{W}(\Delta) \chi_{R}(X)=\frac{z_{\Delta+(|R|-|\Delta|) \cdot 1}}{(|R|-|\Delta|) ! z_{\Delta}} \varphi_{R}(\Delta+(|R|-|\Delta|) \cdot 1) \chi_{R}[X] \stackrel{(43)}{=} \varphi_{R}(\Delta) \chi_{R}[X] .
$$

Последнее равенство тривиально выполняется, когда $\Delta$ не содержит единичных столбцов, $1 \notin \Delta$, в силу того, что в этом случае $z_{\Delta+(|R|-|\Delta|) \cdot 1}=(|R|-|\Delta|) ! z_{\Delta}$ и $\varphi_{R}(\Delta+(|R|-|\Delta|) \cdot 1)=\varphi_{R}(\Delta)$. Если в $\Delta$ имеется $k$ единичных столбцов, то

$$
z_{\Delta+(|R|-|\Delta|) \cdot 1}=\frac{(|R|-|\Delta|+k) !}{k !} z_{\Delta}
$$

в то время как

$$
\varphi_{R}(\Delta+(|R|-|\Delta|) \cdot 1) \stackrel{(43)}{=} \frac{k !(|R|-|\Delta|) !}{(|R|-|\Delta|+k) !} \varphi_{R}(\Delta) .
$$

Возвращаясь к (68), получаем из этого соотношения разложение по характерам произвольного коррелятора $\Psi$-следов. Конечно, эта формула всего лишь полуфабрикат: требуется еще аналог преобразования (58), чтобы превратить ее в более явное 
выражение. K сожалению, корреляторы следов $\Psi$ в любом случае не исчерпывают всех корреляторов в теории ХЧИЗ, а вычисление других корреляторов остается нерешенной, недооцененной и недостаточно исследованной задачей, которой мы посвящаем два отдельных пункта в конце этого обзора.

4.5. Парные корреляторы в теории ХЧИЗ. Вычисление унитарных интегралов с мерой ХЧИЗ стало важной научной задачей с появлением модели КазаковаМигдала для решеточной глюодинамики [27], и первоначально эта задача была рассмотрена в работах [29] и [30], [31]. Если учесть, что интеграл ХЧИЗ (51) имеет дустермаат-хекмановскую природу [5], [14], то становится понятным, что данная задача оказывается также естественным шагом в развитии техники "локализации" и БРСТ-методологии [50]-[53], [59]-[62]. Разумеется, задача связана также с общими исследованиями негауссовых интегралов в теории интегральных дискриминантов [54] - важного раздела бурно развивающейся в последнее время нелинейной алгебры [55]. К сожалению, как уже говорилось, корреляторы ХЧИЗ остаются недооцененной и недостаточно иследованной областью, что не столь уж и удивительно, если учесть бедность наших знаний о самых обычных унитарных интералах, которые были рассмотрены выше в разделе 2. Как бы то ни было, интерес к этой области постепенно возвращается, в частности недавние статьи [14] подтверждают и обобщают старые результаты, полученные в работе [30]. Этот пункт содержит не более чем сжатый обзор современного состояния дел.

В принципе корреляторы можно было бы вычислить напрямую - взяв свою любимую параметризацию унитарных матриц и явно вычислив все $N^{2}$ интегралов. Примером разумной и удобной для этой цели параметризации [31] являются свободные координаты Гельфанда-Цейтлина, на которые не наложены никакие связи. Эти координаты возникают естественным образом в рекурсивной по $N$ процедуре, стартующей с соотношения дуальности (53) и подробно описанной в работах [14]. K сожалению, несмотря на всю свою естественность, подобный подход пока не привел ни к какой общей формуле. Как обычно, более практичным оказался "струнный" подход [61], предлагающий вычислять не отдельные корреляторы, а их производящие функции.

Естественной для теории ХЧИЗ является производящая функция

$$
\begin{aligned}
Z_{\mathrm{IZ}} & =\int e^{\operatorname{tr} X U Y U^{\dagger}+\operatorname{tr} J^{\dagger} U+\operatorname{tr} J U^{\dagger}}[d U] \stackrel{(2)}{\sim} \\
& \stackrel{(2)}{\sim} \int e^{\operatorname{tr} S\left(I-M M^{\dagger}\right)} e^{\operatorname{tr} X M Y M^{\dagger}+\operatorname{tr} J^{\dagger} M+\operatorname{tr} J M^{\dagger}} d^{2} M d S= \\
& =\int e^{\operatorname{tr}_{N^{2} \times N^{2}} J^{\dagger}(S \otimes I-X \otimes Y)^{-1} J} \frac{e^{\operatorname{tr} S} d S}{\operatorname{det}_{N^{2} \times N^{2}}(S \otimes I-X \otimes Y)}= \\
& =\int \exp \left\{\sum_{k}\left(S_{k k}+J_{k i}^{\dagger}\left(S-X y_{k}\right)_{i j}^{-1} J_{j k}\right)\right\} \frac{d S}{\prod_{k} \operatorname{det}\left(S-y_{k} X\right)}
\end{aligned}
$$


как непосредственное обобщение (52) и (53). Интеграл по $M$ гауссов, так что к нему можно применять теорему Вика. Нетривиальным является вычисление интеграла по $S$. Интересно также включить в подынтегральное выражение множитель Лейтвиллера-Смилги $(\operatorname{det} U)^{\nu}$, но (как мы имели случай убедиться в п. 4.3) это приводит к нетривиальным изменениям формул. К сожалению, немногое известно об этой функции даже при $\nu=0$. Не видно никаких препятствий к тому, чтобы рассмотреть что-то более общее, чем первый нетривиальный коррелятор $\left\langle U_{i j} U_{k l}^{\dagger}\right\rangle_{\mathrm{IZ}}$, однако он остается единственным относительно хорошо изученным случаем.

Данный парный коррелятор существенно упрощается, если рассмотреть его в том базисе, где и $X$, и $Y$ диагональны. Тогда, как замечено в работах [29],

$$
\int U_{i j} U_{k l}^{\dagger}[d U]=\mathcal{M}_{i j} \delta_{k j} \delta_{i l}
$$

Согласно работе [30] в этом базисе производящая функция записывается так:

$$
\begin{aligned}
& \sum_{i, j=1}^{N} a_{i} \mathcal{M}_{i j} b_{j}=\sum_{i, j=1}^{N} a_{i} b_{j} \int\left|U_{i j}\right|^{2} \exp \left(\sum_{k, l} x_{k} y_{l}\left|U_{k l}\right|^{2}\right)[d U]= \\
& =\frac{1}{\Delta(X) \Delta(Y)} \sum_{P \in S_{N}}(-1)^{P} e^{x_{i} y_{P(i)}} \sum_{n=0}^{N-1}(-1)^{n} \times \\
& \times \sum_{1 \leqslant i_{1}<\cdots<i_{n+1} \leqslant N} \frac{\operatorname{det}\left(\begin{array}{ccc}
1 & \ldots & 1 \\
x_{i_{1}} & \ldots & x_{i_{n+1}} \\
\vdots & \ddots & \vdots \\
x_{i_{1}}^{n-1} & \ldots & x_{i_{n+1}}^{n-1} \\
a_{i_{1}} & \ldots & a_{i_{n+1}}
\end{array}\right) \operatorname{det}\left(\begin{array}{ccc}
1 & \ldots & 1 \\
y_{P\left(i_{1}\right)} & \ldots & y_{P\left(i_{n+1}\right)} \\
\vdots & \ddots & \vdots \\
y_{P\left(i_{1}\right)}^{n-1} & \cdots & y_{P\left(i_{n+1}\right)}^{n-1} \\
b_{P\left(i_{1}\right)} & \ldots & b_{P\left(i_{n+1}\right)}
\end{array}\right)}{\operatorname{det}\left(\begin{array}{ccc}
1 & \ldots & 1 \\
x_{i_{1}} & \ldots & x_{i_{n+1}} \\
\vdots & \ddots & \vdots \\
x_{i_{1}}^{n} & \ldots & x_{i_{n+1}}^{n}
\end{array}\right) \operatorname{det}\left(\begin{array}{ccc}
1 & \ldots & 1 \\
y_{P\left(i_{1}\right)} & \cdots & y_{P\left(i_{n+1}\right)} \\
\vdots & \ddots & \vdots \\
y_{P\left(i_{1}\right)}^{n} & \cdots & y_{P\left(i_{n+1}\right)}^{n}
\end{array}\right)}= \\
& =\frac{1}{\Delta(X) \Delta(Y)} \sum_{P \in S_{N}}(-1)^{P} e^{x_{i} y_{P(i)}}\left(\sum_{i=1}^{N} a_{i} b_{P(i)}-\sum_{1 \leqslant i<j \leqslant N} \frac{\left(a_{i}-a_{j}\right)\left(b_{P(i)}-b_{P(j)}\right)}{\left(x_{i}-x_{j}\right)\left(y_{P(i)}-y_{P(j)}\right)}+\right. \\
& \left.+\sum_{1 \leqslant i<j<k \leqslant N} \frac{\left|\begin{array}{ccc}
1 & 1 & 1 \\
x_{i} & x_{j} & x_{k} \\
a_{i} & a_{j} & a_{k}
\end{array}\right|\left|\begin{array}{ccc}
1 & 1 & 1 \\
y_{P(i)} & y_{P(j)} & y_{P(k)} \\
b_{P(i)} & b_{P(j)} & b_{P(k)}
\end{array}\right|}{\left(x_{i}-x_{j}\right)\left(x_{j}-x_{k}\right)\left(x_{i}-x_{k}\right)\left(y_{P(i)}-y_{P(j)}\right)\left(y_{P(j)}-y_{P(k)}\right)\left(y_{P(i)}-y_{P(k)}\right)}+\cdots\right) .
\end{aligned}
$$


В работах [14] эта громоздкая формула была преобразована к более изящному виду

$$
\mathcal{M}_{i j}=\left.\frac{1}{\Delta(X) \Delta(Y)} \operatorname{res}\left\{\operatorname{det} E-\operatorname{det}\left(E-\frac{1}{X-u \cdot I} E \frac{1}{Y-v \cdot I}\right)\right\}\right|_{\substack{u=x_{i} \\ v=y_{j}}},
$$

где матрица $E$ определена так: $E_{i j}=e^{x_{i} y_{j}}$. При использовании этой формулы вычетов надо взять коэффициент перед $\left(\left(u-x_{i}\right)\left(v-y_{j}\right)\right)^{-1}$ в сумме по перестановкам

$$
\sum_{P \in S_{N}}(-1)^{P} \prod_{k} e^{x_{k} y_{P(k)}}\left(1-\frac{1}{\left(u-x_{k}\right)\left(v-y_{P(k)}\right)}\right) .
$$

Первый член $\operatorname{det} E$ в $(75)$ не дает вклада в вычет. Фактически возникают два разных распределения: одно для перестановок $P$ со свойством $j=P(i)$ и другое для всех остальных перестановок:

$$
\begin{aligned}
\mathcal{M}_{i j}= & \frac{1}{\Delta(X) \Delta(Y)} \sum_{P \in S_{N}} \sum_{P}(-1)^{P}\left\{\delta_{P(i), j} \prod_{k \neq i}^{N}\left(1-\frac{1}{\left(x_{i}-x_{k}\right)\left(y_{j}-y_{P(k)}\right)}\right)-\right. \\
& -\left(1-\delta_{P(i), j}\right) \frac{1}{\left(x_{i}-x_{P(j)}\right)\left(y_{j}-y_{P(i)}\right)} \times \\
& \left.\times \prod_{k \neq i, P^{-1}(j)}^{N}\left(1-\frac{1}{\left(x_{i}-x_{k}\right)\left(y_{j}-y_{P(k)}\right)}\right)\right\} \prod_{k=1}^{N} e^{x_{k} y_{P(k)}} .
\end{aligned}
$$

В частности, при $N=1$ остается только один член первого типа, и $\mathcal{M}_{11}=e^{x_{1} y_{1}}$.

При $N=2$ вклад дают члены обоих типов:

$$
\begin{aligned}
& \mathcal{M}_{11}=\frac{1}{x_{12} y_{12}}\left\{\left(1-\frac{1}{x_{12} y_{12}}\right) e^{x_{1} y_{1}+x_{2} y_{2}}+\frac{1}{x_{12} y_{12}} e^{x_{1} y_{2}+x_{2} y_{1}}\right\}=\mathcal{M}_{22}, \\
& \mathcal{M}_{12}=\frac{1}{x_{12} y_{12}}\left\{\frac{1}{x_{12} y_{12}} e^{x_{1} y_{1}+x_{2} y_{2}}-\left(1+\frac{1}{x_{12} y_{12}}\right) e^{x_{1} y_{2}+x_{2} y_{1}}\right\}=\mathcal{M}_{21},
\end{aligned}
$$

что в точности воспроизводит (74), где при $N=2$ выживают только два первых члена в правой части. Конечно, $\mathcal{M}_{11}+\mathcal{M}_{12}=J_{\mathrm{IZ}}$.

В общем случае эквивалентность (74) и (75) следует из того, что для $a_{i}=1 /\left(u-x_{i}\right)$ определители в знаменателе формулы (74) обращаются в

$$
\operatorname{det}\left(\begin{array}{ccc}
1 & \cdots & 1 \\
x_{i_{1}} & \cdots & x_{i_{n+1}} \\
\vdots & \ddots & \vdots \\
x_{i_{1}}^{n-1} & \ldots & x_{i_{n+1}^{n-1}}^{n-1} \\
\left(u-x_{i_{1}}\right)^{-1} & \ldots & \left(u-x_{i_{n+1}}\right)^{-1}
\end{array}\right)=\operatorname{det}\left(\begin{array}{ccc}
1 & \ldots & 1 \\
x_{i_{1}} & \cdots & x_{i_{n+1}} \\
\vdots & \ddots & \vdots \\
x_{i_{1}}^{n} & \cdots & x_{i_{n+1}}^{n}
\end{array}\right) \prod_{k=1}^{n+1} \frac{1}{u-x_{i_{k}}}
$$


просто потому, что знаменатель имеет размерность $n(n-1) / 2$ и при этом антисимметричен по всем $x_{i_{k}}$. Поэтому из (74) следует, что

$$
\begin{aligned}
& \sum_{i, j} \frac{1}{u-x_{i}} \mathcal{M}_{i j} \frac{1}{v-y_{j}}= \\
& =\frac{1}{\Delta(X) \Delta(Y)} \sum_{P \in S_{N}}(-1)^{P} e^{x_{i} y_{P(i)}}\left\{\sum_{n=0}^{N-1}(-1)^{n} \sum_{\substack{1 \leqslant i_{1}<\ldots, \cdots<i_{n+1} \leqslant N}} \prod_{k=1}^{n+1} \frac{1}{\left(u-x_{i_{k}}\right)\left(v-y_{P\left(i_{k}\right)}\right)}\right\}= \\
& =\frac{1}{\Delta(X) \Delta(Y)} \sum_{P \in S_{N}}(-1)^{P} e^{x_{i} y_{P(i)}}\left\{1-\prod_{i=1}^{N}\left(1-\frac{1}{\left(u-x_{i}\right)\left(v-y_{P(i)}\right)}\right)\right\}= \\
& =\frac{\operatorname{det} E-\operatorname{det}\left(E-\frac{1}{u-X} E \frac{1}{v-Y}\right)}{\Delta(X) \Delta(Y)} .
\end{aligned}
$$

Отметим, что предельный переход от этой формулы к формуле (4) является непростым упражнением, и это не так уж удивительно, поскольку даже свойство $J_{\mathrm{IZ}}(X, Y)=1$ при $X, Y=0$ в формуле (47) является не вполне очевидным. Гораздо проще проверить, что соотношение (80) совместно с равенством $\sum_{j}\left|U_{i j}\right|^{2}=1$, т.е. что $\sum_{j} \mathcal{M}_{i j}=J_{\text {IZ }}$ для любого $i$. В самом деле,

$$
\begin{aligned}
\sum_{i, j} \frac{1}{u-x_{i}} \mathcal{M}_{i j} & =\sum_{j} \oint_{y_{j}} \frac{\operatorname{det} E-\operatorname{det}\left(E-\frac{1}{u-X} E \frac{1}{v-Y}\right)}{\Delta(X) \Delta(Y)} d v= \\
& =\frac{\operatorname{det} E}{\Delta(X) \Delta(Y)} \oint_{\infty}\left(1-\operatorname{det}\left(1-\frac{1}{u-X} E \frac{1}{v-Y} E^{-1}\right)\right) d v= \\
& =J_{\text {IZ }} \oint_{\infty} \operatorname{tr} \frac{1}{u-X} E \frac{1}{v-Y} E^{-1} d v=J_{\text {IZ }} \operatorname{tr} \frac{1}{u-X} .
\end{aligned}
$$

Разложение парного коррелятора по характерам можно получить по аналогии с (54), если известно подходящее обобщение (16) и (17), а именно

$$
\int \mathcal{U}_{R} \mathcal{U}_{R}^{\dagger} \mathcal{U}_{R^{\prime}} \mathcal{U}_{R^{\prime}}^{\dagger}[d U]
$$

- обощение (5) на произвольные представления. Для разложения производящей функции (74) фактически достаточно случая, когда $R^{\prime}$ - первое фундаментальное представление.

Как и сам интеграл ХЧИЗ, парный коррелятор удовлетворяет соотношению типа дуальности - прямому обобщению формулы (53): из того, что

$$
\int U_{i j} U_{k l}^{\dagger}[d U] \stackrel{(72)}{\sim} \delta_{j k} \int\left(\frac{1}{S-X y_{k}}\right)_{i l}^{-1} \frac{e^{\operatorname{tr} S} d S}{\prod_{k} \operatorname{det}\left(S-X y_{k}\right)},
$$

после замены матричных переменных интегрирования $S \longrightarrow X S$ и после диагонализации матриц $X$ и $S$ следует, что

$$
\mathcal{M}_{i j}(X, Y) \sim \int \frac{\mathcal{M}_{i j}(X, S) \Delta^{2}(S) \prod_{p} d s_{p}}{\left(s_{i}-y_{j}\right) x_{j} \prod_{p, q}\left(s_{p}-y_{q}\right)} .
$$


Непосредственно из (72) можно вывести сходные соотношения и для старших корреляторов в теории ХЧИЗ. Об обобщениях на другие простые группы Ли см., например, работы [7] и [14].

4.6. Пример $U(2)$. Чтобы проиллюстрировать использование соотношений дуальности, мы явно покажем, как функции (74) и (75) удовлетворяют (83) в простейшем содержательном случае $N=2$.

Начнем с еще более простого случая - соотношения дуальности (53) при $N=1$. Утверждение тогда сводится к равенству

$$
e^{x y}=\oint \frac{e^{x s} d s}{s-y}
$$

и понятно, что интеграл следует понимать как вычет в полюсе при $s=y$ (как обычно, множитель $2 \pi i$ включен в определение контурного интеграла).

При $N=2$ то же соотношение (53) становится более сложным:

$$
\begin{aligned}
& \frac{e^{x_{1} y_{1}+x_{2} y_{2}}-e^{x_{1} y_{2}+x_{2} y_{1}}}{x_{12} y_{12}} \stackrel{(72)}{\sim} \oint \oint \frac{e^{x_{1} s_{1}+x_{2} s_{2}}-e^{x_{1} s_{2}+x_{2} s_{1}}}{x_{12} s_{12}} \times \\
& \quad \times \frac{s_{12}^{2} d s_{1} d s_{2}}{\left(s_{1}-y_{1}\right)\left(s_{1}-y_{2}\right)\left(s_{2}-y_{1}\right)\left(s_{2}-y_{2}\right)}= \\
&=\frac{1}{x_{12} y_{12}^{2}} \oint \oint\left(e^{x_{1} s_{1}+x_{2} s_{2}}-e^{x_{1} s_{2}+x_{2} s_{1}}\right)\left(\frac{1}{s_{1}-y_{1}}-\frac{1}{s_{1}-y_{2}}\right) \times \\
& \quad \times\left(\frac{1}{s_{2}-y_{1}}-\frac{1}{s_{2}-y_{2}}\right) s_{12} d s_{1} d s_{2}= \\
&=\frac{1}{x_{12} y_{12}^{2}}\left(-y_{12}\left(e^{x_{1} y_{1}+x_{2} y_{2}}-e^{x_{1} y_{2}+x_{2} y_{1}}\right)-y_{21}\left(e^{x_{1} y_{2}+x_{2} y_{1}}-e^{x_{1} y_{1}+x_{2} y_{2}}\right)\right)= \\
&=-2 \frac{e^{x_{1} y_{1}+x_{2} y_{2}}-e^{x_{1} y_{2}+x_{2} y_{1}}}{x_{12} y_{12}} .
\end{aligned}
$$

Заметим, что из четырех возможных полюсов $\left(s_{1}, s_{2}\right)=\left(y_{1}, y_{1}\right),\left(y_{1}, y_{2}\right),\left(y_{2}, y_{1}\right)$, $\left(y_{2}, y_{2}\right)$ первый и последний не дают вклада из-за множителя $s_{12}=s_{1}-s_{2}$ в числителе подынтегрального выражения.

Возвращаемся к тождеству (83). В случае $N=1$ в нем просто утверждается, что

$$
e^{x y}=\oint \frac{e^{x s} d s}{x(s-y)^{2}}=\left.\frac{1}{x} \frac{\partial e^{x s}}{\partial s}\right|_{s=y}=e^{x y} .
$$

При $N=2$ вычисление существенно удлиняется. Мы приведем его только для одного (из четырех) ненулевых корреляторов (78), а именно для

$$
\mathcal{M}_{11}(X, Y)=-\frac{1}{x_{12}^{2} y_{12}^{2}}\left(\left(1-x_{12} y_{12}\right) e^{x_{1} y_{1}+x_{2} y_{2}}-e^{x_{1} y_{2}+x_{2} y_{1}}\right) .
$$


С помощью тех же приемов, что использовались в (85), можно переписать правую часть (83) как

$$
\begin{aligned}
& \oint \oint \frac{s_{12}^{2} d s_{1} d s_{2}}{\left(s_{1}-y_{1}\right) x_{1} y_{12}^{2}}\left(\frac{1}{s_{1}-y_{1}}-\frac{1}{s_{1}-y_{2}}\right)\left(\frac{1}{s_{2}-y_{1}}-\frac{1}{s_{2}-y_{2}}\right) \times \\
& \quad \times\left\{-\left(\frac{1}{x_{12}^{2}}-\frac{s_{12}}{x_{12}}\right) e^{x_{1} s_{1}+x_{2} s_{2}}+\frac{1}{x_{12}^{2}} e^{x_{1} s_{2}+x_{2} s_{1}}\right\}= \\
&=\frac{1}{x_{1} x_{12}^{2} y_{12}^{2}} \oint \oint\left\{-\frac{1}{\left(s_{1}-y_{1}\right)^{2}}+\frac{1}{y_{12}}\left(\frac{1}{s_{1}-y_{1}}-\frac{1}{s_{1}-y_{2}}\right)\right\} \times \\
& \quad \times\left(\frac{1}{s_{2}-y_{1}}-\frac{1}{s_{2}-y_{2}}\right)\left(\left(1-s_{12} x_{12}\right) e^{x_{1} s_{1}+x_{2} s_{2}}-e^{x_{1} s_{2}+x_{2} s_{1}}\right) d s_{1} d s_{2} .
\end{aligned}
$$

Для облегчения анализа этой и последующих формул мы подчеркиваем члены, происходящие из двойных полюсов при $s_{1}=y_{1}$ и порождающие производные подынтегрального выражения в интеграле по $s_{1}$. На этот раз все четыре полюса могут давать вклад, и сокращение двух нежелательных вкладов несколько менее тривиально: для $\left(s_{1}, s_{2}\right)=\left(y_{1}, y_{1}\right)$ мы получаем

$$
\left(\underline{-\left(x_{1}-x_{12}\right)+x_{2}}+\frac{1}{y_{12}}(1-1)\right) e^{\left(x_{1}+x_{2}\right) y_{1}}=0
$$

а для $\left(s_{1}, s_{2}\right)=\left(y_{2}, y_{2}\right)-$

$$
\frac{1}{y_{12}}(1-1) e^{\left(x_{1}+x_{2}\right) y_{2}}=0 .
$$

Неисчезающий вклад дают два других полюса: для $\left(s_{1}, s_{2}\right)=\left(y_{2}, y_{1}\right)$ имеем

$$
-\frac{1}{y_{12}}\left(\left(1+x_{12} y_{12}\right) e^{x_{1} y_{2}+x_{2} y_{1}}-e^{x_{1} y_{1}+x_{2} y_{2}}\right),
$$

наконец, для $\left(s_{1}, s_{2}\right)=\left(y_{1}, y_{2}\right)$ имеем

$$
\begin{aligned}
& -\frac{1}{y_{12}}\left(\left(1-x_{12} y_{12}\right) e^{x_{1} y_{1}+x_{2} y_{2}}-e^{x_{1} y_{2}+x_{2} y_{1}}\right)+ \\
& +\underline{\left(x_{1}\left(1-x_{12} y_{12}\right)-x_{12}\right) e^{x_{1} y_{1}+x_{2} y_{2}}-x_{2} e^{x_{1} y_{2}+x_{2} y_{1}}} .
\end{aligned}
$$

Собирая все выражения, получаем для правой части (83)

$$
\begin{gathered}
\frac{1}{x_{1} x_{12}^{2} y_{12}^{2}}\left\{\left(\frac{1}{y_{12}}+\underline{x_{1}-x_{12}-x_{1} x_{12} y_{12}}-\frac{1}{y_{12}}+x_{12}\right) e^{x_{1} y_{1}+x_{2} y_{2}}+\right. \\
+\left(-\frac{1}{y_{12}}-x_{12}-x_{2}\right. \\
\left.\left.+\frac{1}{y_{12}}\right) e^{x_{1} y_{2}+x_{2} y_{1}}\right\}= \\
=\frac{1}{x_{12}^{2} y_{12}^{2}}\left\{\left(1-x_{12} y_{12}\right) e^{x_{1} y_{1}+x_{2} y_{2}}-e^{x_{1} y_{2}+x_{2} y_{1}}\right\}=-\mathcal{M}_{11}(X, Y) .
\end{gathered}
$$


Чтобы дать некоторое представление о том, на что похожи старшие корреляторы, приведем здесь ненулевые четырехточечные корреляторы при $N=2$ :

$$
\begin{aligned}
\int U_{11}^{2}\left(U_{11}^{\dagger}\right)^{2}[d U] & =\frac{1}{x_{12}^{3} y_{12}^{3}}\left\{\left(2-2 x_{12} y_{12}+x_{12}^{2} y_{12}^{2}\right) e^{x_{1} y_{1}+x_{2} y_{2}}-2 e^{x_{1} y_{2}+x_{2} y_{1}}\right\}, \\
\int U_{11} U_{12} U_{11}^{\dagger} U_{21}^{\dagger}[d U] & =\frac{1}{x_{12}^{3} y_{12}^{3}}\left\{\left(-2+x_{12} y_{12}\right) e^{x_{1} y_{1}+x_{2} y_{2}}+\left(2+x_{12} y_{12}\right) e^{x_{1} y_{2}+x_{2} y_{1}}\right\}, \\
\int U_{11} U_{22} U_{11}^{\dagger} U_{22}^{\dagger}[d U] & =\frac{1}{x_{12}^{3} y_{12}^{3}}\left\{\left(2-2 x_{12} y_{12}+x_{12}^{2} y_{12}^{2}\right) e^{x_{1} y_{1}+x_{2} y_{2}}-2 e^{x_{1} y_{2}+x_{2} y_{1}}\right\}, \\
\int U_{11} U_{22} U_{12}^{\dagger} U_{21}^{\dagger}[d U] & =\frac{1}{x_{12}^{3} y_{12}^{3}}\left\{\left(2-x_{12} y_{12}\right) e^{x_{1} y_{1}+x_{2} y_{2}}-\left(2+x_{12} y_{12}\right) e^{x_{1} y_{2}+x_{2} y_{1}}\right\}, \\
\int U_{12}^{2}\left(U_{21}^{\dagger}\right)^{2}[d U] & =\frac{1}{x_{12}^{3} y_{12}^{3}}\left\{2 e^{x_{1} y_{1}+x_{2} y_{2}}-\left(2+2 x_{12} y_{12}+x_{12}^{2} y_{12}^{2}\right) e^{x_{1} y_{2}+x_{2} y_{1}}\right\}, \\
\int U_{12} U_{21} U_{12}^{\dagger} U_{21}^{\dagger}[d U] & =\frac{1}{x_{12}^{3} y_{12}^{3}}\left\{2 e^{x_{1} y_{1}+x_{2} y_{2}}-\left(2+2 x_{12} y_{12}+x_{12}^{2} y_{12}^{2}\right) e^{x_{1} y_{2}+x_{2} y_{1}}\right\} .
\end{aligned}
$$

Другие неисчезающие корреляторы получаются действием дискретных симметрий $1 \leftrightarrow 2$, например

$$
\int U_{22}^{2}\left(U_{22}^{\dagger}\right)^{2}[d U]=\int U_{11}^{2}\left(U_{11}^{\dagger}\right)^{2}[d U]
$$

Легко проверить, что сумма первого и последнего равенств в (89), которая должна равняться $\int U_{11} U_{11}^{\dagger}[d U]$, действительно воспроизводит $\mathcal{M}_{11}$ из (85). Точно так же сумма второго и пятого равенств воспроизводит $\mathcal{M}_{12}$, в то время как сумма второго и четвертого равенств должна обращаться в нуль - и она действительно равна нулю. Далее, в пределе $x, y \rightarrow 0$ выражения (89) превращаются в (5), хотя, как уже говорилось, этот предел не так легко взять: даже в этой простой ситуации $(N=2)$ приходится разлагать экспоненты до третьей степени по их аргументам.

Последний критерий существенно ограничивает возможную форму $2 m$-точечного коррелятора:

$$
\int U^{\otimes m}\left(U^{\dagger}\right)^{\otimes m}[d U]=\frac{1}{\left(x_{12} y_{12}\right)^{m+1}}\left(P\left(x_{12} y_{12}\right) e^{x_{1} y_{1}+x_{2} y_{2}}-Q\left(x_{12} y_{12}\right) e^{x_{1} y_{2}+x_{2} y_{1}}\right)
$$

где $P(t)=\sum_{k=0}^{m} P_{k} t^{k}$ и $Q(t)=\sum_{k=0}^{m} Q_{k} t^{k}-$ многочлены степени $m$ по переменной $t=x_{12} y_{12}=\left(x_{1}-x_{2}\right)\left(y_{1}-y_{2}\right)$. Условие, что эти выражения несингулярны при $t=0$, подразумевает, что

$$
P_{k}=\sum_{j=0}^{k} \frac{(-1)^{j}}{j !} Q_{k-j}
$$


т.е. многочлен $P(t)$ полностью определен, если известен $Q(t)$. Выбор $Q(t)$ зависит от коррелятора. В частности,

$$
\int U_{11}^{m}\left(U_{11}^{\dagger}\right)^{m}[d U]=\frac{(-1)^{m}}{\left(x_{12} y_{12}\right)^{m+1}}\left(e^{x_{1} y_{1}+x_{2} y_{2}} \sum_{k=0}^{m} \frac{(-1)^{k} m !}{k !}\left(x_{12} y_{12}\right)^{k}-e^{x_{1} y_{2}+x_{2} y_{1}} m !\right) .
$$

Из этого примера ясно, что обобщение соотношений $(74),(75)$ на четырехточечные и более высокие корреляторы должно быть прямым и непосредственным. Его, однако, еще только предстоит найти.

Благодарности. Я признателен А. Александрову, А. Миронову, А. Пополитову и Ш. Шакирову за сотрудничество и помощь. Работа частично поддержана Государственной корпорацией по атомной энергии "РосАтом", Программой поддержки ведущих научных школ (грант НШ-3035.2008.2) и РФФИ (гранты № 07-02-00645, 09-02-91005-ANF, 09-02-90493-Ukr, 09-02-93105-CNRSL и 09-01-92440-CE).

\section{Список литературы}

[1] E. P. Wigner, Ann. Math., 62:3 (1955), 548-564; F. J. Dyson, J. Math. Phys., 3:1 (1962), 140, 157, 166, 1191, 1199; E. Brézin, C. Itzykson, G. Parisi, J. B. Zuber, Comm. Math. Phys., 59:1 (1978), 35-51; D. Bessis, Comm. Math. Phys., 69:2 (1979), 147-163; D. Bessis, C. Itzykson, J. B. Zuber, Adv. Appl. Math., 1:2 (1980), 109-157; M. L. Mehta, Comm. Math. Phys., 79:3 (1981), 327-340; Random Matrices, Acad. Press, Boston, MA, 1991; D. Bessis, C. Itzykson, J.-B. Zuber, Adv. Appl. Math., 1:2 (1980), 109-157; A. A. Migdal, Phys. Rep., 102:4 (1983), 199-290; F. David, Nucl. Phys. B, 257:1 (1985), 45-58; 543-576; J. Ambjørn, B. Durhuus, J. Fröhlich, Nucl. Phys. B, 257:3 (1985), 433-449; V. A. Kazakov, I. K. Kostov, A. A. Migdal, Phys. Lett. B, 157:4 (1985), 295-300; D. V. Boulatov, V.A. Kazakov, I. K. Kostov, A. A. Migdal, Phys. Lett. B, 174:1 (1986), 87-93; Nucl. Phys. B, 275:4 (1986), 641-686; V. A. Kazakov, Phys. Lett. A, 119:3 (1986), 140-144; Modern Phys. Lett. A, 4:22 (1989), 2125-2139; E. Brézin, V. Kazakov, Phys. Lett. B, 236:2 (1990), 144-150; D. J. Gross, A. A. Migdal, Phys. Rev. Lett., 64:2 (1990), 127-130; Nucl. Phys. B, 340:2-3 (1990), 333-365; M. Douglas, S. Shenker, Nucl. Phys. B, 335:3 (1990), 635-654; L. Alvarez-Gaumé, "Random surfaces, statistical mechanics and string theory", Helv. Phys. Acta, 64:4 (1991), 359-526; M. R. Douglas, Phys. Lett. B, 238:2-4 (1990), 176-180; A. Levin, A. Morozov, Phys. Lett. B, 243:3 (1990), 207-214; P. Ginsparg, Matrix Models of $2 d$ Gravity, arXiv: hep-th/9112013; D. J. Gross, M. J. Newmann, Phys. Lett. B, 266:3-4 (1991), 291-297; A. Marshakov, A. Mironov, A. Morozov, Phys. Lett. B, 265:1-2 (1991), 99-107; R. Dijkgraaf, G. Moore, R. Plesser, Nucl. Phys. B, 394:2 (1993), 356-382; arXiv: hep-th/9208031; J.-M. Daul, V. A. Kazakov, I. K. Kostov, Nucl. Phys. B, 409:2 (1993), 311-338; M. Staudacher, Phys. Lett. B, 305:4 (1993), 332-338; arXiv: hep-th/9301038; J. Ambjørn, L. Chekhov, C. F. Kristjansen, Yu. Makeenko, Nucl. Phys. B, 404:1-2 (1993), 127-172; Erratum, 449 (1995), 681; arXiv: hep-th/9302014; P. Di Francesco, P. Ginsparg, J. Zinn-Justin, Phys. Rep., 254:1-2 (1995), 1-133; arXiv: hep-th/9306153; A. Mironov, Internat. J. Modern Phys. A, 9:25 (1994), 4355-4405; arXiv: hep-th/9312212; ЯФ, 33 (2002), 1051-1145; B. Eynard, Large random matrices: eigenvalue distribution, arXiv: hep-th/9401165; M. Adler, A. Morozov, T. Shiota, P. van Moerbeke, Nucl. Phys. Proc. Suppl., 49:1-3 (1996), 201-212; arXiv: hep-th/9603066;

2 Теоретическая и математическая физика, т. 162, № 1, 2010 г. 
G. Akemann, Nucl. Phys. B, 482:1-2 (1996), 403-430; arXiv: hep-th/9606004; G. Akemann, P. H. Damgaard, U. Magnea, S. Nishigaki, Nucl. Phys. B, 487:3 (1997), 721-738; 519:3 (1998), 682-714; arXiv: hep-th/9609174; arXiv: hep-th/9712006; J. Ambjørn, M. G. Harris, M. Weis, Nucl. Phys. B, 504:1-2 (1997), 482-510; arXiv: hep-th/9702188; A. Marshakov, M. Martellini, A. Morozov, Phys. Lett. B, 418:3-4 (1998), 294-302; arXiv: hep-th/9706050; T. Guhr, A. Mueller-Groeling, H. A. Weidenmüller, Phys. Rep., 299:4-6 (1998), 189-425; arXiv: cond-mat/9707301; V. A. Kazakov, I. K. Kostov, N. A. Nekrasov, Nucl. Phys. B, 557:3 (1999), 413-442; arXiv: hep-th/9810035; M. Adler, P. van Moerbecke, Ann. Math., 149:3 (1999), 921-976; B. Eynard, Random Matrices. Saclay Lecture Notes, 2000, http://ipht.cea.fr/Docspht//articles/t01/014/public/publi.pdf; A. Gerasimov, A. Morozov, K. Selivanov, Internat. J. Modern Phys. A, 16:9 (2001), 1531-1558; arXiv: hep-th/0005053; A. Okounkov, Int. Math. Res. Not., 2002:18 (2002), 933-957; arXiv: math.AG/0101201; P. Ormerod, C. Mounfield, Physica A, 280:3-4 (2000), 497-504; arXiv: cond-mat/0102357; P. Di Francesco, Nucl. Phys. B, 648:3 (2003), 461-496; arXiv: cond-mat/0208037; P. J. Forrester, N. C. Snaith, J. J. M. Verbaarschot, J. Phys. A, 36:12 (2003), R1-R10; arXiv: cond-mat/0303207; S. Alexandrov, V. Kazakov, D. Kutasov, JHEP, 09 (2003), 057; arXiv: hep-th/0306177; N. Seiberg, D. Shih, JHEP, 02 (2004), 021; arXiv: hep-th/0312170; V. Kazakov, I. Kostov, "Instantons in non-critical strings from the two-matrix model", From Fields to Strings: Circumnavigating Theoretical Physics, V. 3, eds. M. Shifman, A. Vainshtein J. Wheater, World Sci. Publ., Singapore, 2005, 1864-1894; arXiv: hep-th/0403152; G. Akemann, Y. V. Fyodorov, G. Vernizzi, Nucl. Phys. B, 694:1-2 (2004), 59-98; arXiv: hep-th/0404063; P. Di Francesco, "2D quantum gravity, matrix models and graph combinatorics", Applications of Random Matrices in Physics, NATO Sci. Ser. II Math. Phys. Chem., 221, eds. É. Brézin, V. Kazakov, Didina Serban, P. Wiegmann A. Zabrodin, Springer, Dordrecht, 2006, 33-88; arXiv: math-ph/0406013; A. Morozov, "Challenges of matrix models", String Theory: From Gauge Interactions to Cosmology, NATO Sci. Ser. II Math. Phys. Chem., 208, eds. L. Baulieu, J. de Boer, B. Pioline, E. Rabinovici, Springer, Dordrecht, 2006, 129-162; arXiv: hep-th/0502010; M. Stephanov, J. J. M. Verbaarschot, T. Wettig, Random matrices, arXiv: hep-ph/0509286; Y.V. Fyodorov, B. A. Khoruzhenko, Comm. Math. Phys., 273:3 (2007), 561-599; arXiv: math-ph/0602032; Y. V. Fyodorov, Int. Math. Res. Not., 2006, 61570; arXiv: math-ph/0602039; J. Harnad, A. Yu. Orlov, Physica D, 235:1-2 (2007), 168-206; arXiv: 0704.1157.

[2] А. А. Мигдал, ЖЖЭТФ, 69 (1975), 810-822; C. Itzykson, J.-B. Zuber, Comm. Math. Phys., 134:1 (1990), 197-207; P. Di Francesco, C. Itzykson, Ann. Inst. H. Poincaré, 59:2 (1993), 117-139; V.A. Kazakov, M. Staudacher, T. Wynter, Comm. Math. Phys., 179:1 (1996), 235-256; I. K. Kostov, M. Staudacher, T. Wynter, Comm. Math. Phys., 191:2 (1998), 283-298; arXiv: hep-th/9703189.

[3] V. Kazakov, "Solvable matrix models", Random Matrix Models and Their Applications, Math. Sci. Res. Inst. Publ., 40, eds. P. Bleher, A. Its, Cambridge Univ. Press, Cambridge, 2001, 271-283; arXiv: hep-th/0003064.

[4] А. Ю. Морозов, УФН, 164:1 (1994), 3-62; arXiv: hep-th/9303139.

[5] A. Morozov, "Matrix models as integrable systems", Particles and Fields, CRM Ser. Math. Phys., eds. G. Semenoff, L. Vinet, Springer, New York, 1999, 127-210; arXiv: hep-th/9502091.

[6] F. Cachazo, K. Intriligator, C. Vafa, Nucl. Phys. B, 603:1-2 (2001), 3-41; arXiv: hep-th/0103067; F. Cachazo, C. Vafa, $N=1$ and $N=2$ geometry from fluxes, arXiv: hep-th/0206017; R. Dijkgraaf, C. Vafa, Nucl. Phys. B, 644:1-2 (2002), 3-20; 21-39; arXiv: hep-th/0206255; arXiv: hep-th/0207106; A Perturbative window into non-perturbative physics, arXiv: hep-th/0208048; L. Chekhov, A. Mironov, Phys. Lett. B, 
552:3-4 (2003), 293-302; arXiv: hep-th/0209085; R. Dijkgraaf, S. Gukov, V. A. Kazakov, C. Vafa, Phys. Rev. D, 68:4 (2003), 045007; arXiv: hep-th/0210238; V. A. Kazakov, A. Marshakov, J. Phys. A, 36:12 (2003), 3107-3136; arXiv: hep-th/0211236; H. Itoyama, A. Morozov, Nucl. Phys. B, 657:1-3 (2003), 53-78; arXiv: hep-th/0211245; Phys. Lett. B, 555:3-4 (2003), 287-295; arXiv: hep-th/0211259; Progr. Theor. Phys., 109:3 (2003), 433-463; arXiv: hep-th/0212032; Internat. J. Modern Phys. A, 18:32 (2003), 5889-5906; arXiv: hep-th/0301136; S. G. Naculich, H. Schnitzer, N. Wyllard, JHEP, 01 (2003), 015; arXiv: hep-th/0211254; B. Feng, Nucl. Phys. B, 661:1-2 (2003), 113-138; arXiv: hep-th/0212010; I. Bena, S. de Haro, R. Roiban, Nucl. Phys. B, 664:1-2 (2003), 45-58; arXiv: hep-th/0212083; Ch. Ahn, Phys. Lett. B, 560:1-2 (2003), 116-127; arXiv: hep-th/0301011; L. Chekhov, A. Marshakov, A. Mironov, D. Vasiliev, Phys.Lett. B, 562:3-4 (2003), 323-338; arXiv: hep-th/0301071; Л. О. Чехов, А. В. Маршаков, А. Д. Миронов, Д. Васильев, "Комплексная геометрия матричных моделей”, Нелинейная динамика, Сборник статей, Тр. МИАН, 251, Наука, М., 2005, 265-306; arXiv: hep-th/0506075; A. Dymarsky, V. Pestun, Phys. Rev. D, 67:12 (2003), 125001; arXiv: hep-th/0301135; Yu. Ookouchi, Yo. Watabiki, Modern Phys. Lett. A, 18:16 (2003), 1113-1126; arXiv: hep-th/0301226; H. Itoyama, H. Kanno, Phys. Lett. B, 573:1-4 (2003), 227-234; arXiv: hep-th/0304184; Nucl. Phys. B, 686:1-2 (2004), 155-164; arXiv: hep-th/0312306; M. Matone, L. Mazzucato, JHEP, 07 (2003), 015; arXiv: hep-th/0305225; R. Argurio, G. Ferretti, R. Heise, Internat. J. Modern Phys. A, 19:13 (2004), 2015-2078; arXiv: hep-th/0311066; M. Gómez-Reino, JHEP, 06 (2004), 051; arXiv: hep-th/0405242; K. Fujiwara, H. Itoyama, M. Sakaguchi, Progr. Theor. Phys., 113:2 (2005), 429-455; arXiv: hep-th/0409060; Nucl. Phys. B, 723:1-2 (2005), 33-52; arXiv: hep-th/0503113; Progr. Theor. Phys. Suppl., no. 164 (2007), 125-137; arXiv: hep-th/0602267; Sh. Aoyama, JHEP, 10 (2005), 032; arXiv: hep-th/0504162; D. Berenstein, S. Pinansky, Nucl. Phys. B, 753:1-2 (2006), 69-91; arXiv: hep-th/0602294.

[7] E. Brezin, S. Hikami, An extension of the HarishChandra-Itzykson-Zuber integral, arXiv: math-ph/0208002.

[8] A. Zabrodin, Modern Phys. Lett. A, 7:5 (1992), 441-446; Ann. Henri Poincaré, 4, Suppl. 2, 851-861; arXiv: cond-mat/0210331; P. Wiegmann, A. Zabrodin, "Large $N$ expansion for normal and complex matrix ensembles", Frontiers in Number Theory, Physics, and Geometry, I, eds. P. Cartier, B. Julia, P. Moussa, P. Vanhove, Springer, Berlin, 2006, 213-229; arXiv: hep-th/0309253; R. Teodorescu, E. Bettelheim, O. Agam, A. Zabrodin, P. Wiegmann, Nucl. Phys. B, 704:3 (2005), 407-444; arXiv: hep-th/0401165.

[9] A.Alexandrov, A. Mironov, A. Morozov, Internat. J. Modern Phys. A, 19:24 (2004), 4127-4163; А. С. Александров, А. Д. Миронов, А. Ю. Морозов, ТМФ, 142:3 (2005), 419-488; arXiv: hep-th/0310113.

[10] T. J. Hollowood, A.Iqbal, C. Vafa, JHEP, 03 (2008), 069; arXiv: hep-th/0310272.

[11] A. Alexandrov, A. Mironov, A. Morozov, Internat. J. Modern Phys. A, 21:12 (2006), 2481-2517; arXiv: hep-th/0412099; Fortschr. Phys., 53:5-6 (2005), 512-521; arXiv: hep-th/0412205; A. Alexandrov, A. Mironov, A. Morozov, P. Putrov, Partition functions of matrix models as the first special functions of string theory. II. Kontsevich model, arXiv: 0811.2825.

[12] B. Eynard, JHEP, 11 (2004), 031; arXiv: hep-th/0407261; B. Eynard, N. Orantin, JHEP, 12 (2005), 034; arXiv:math-ph/0504058; Commun. Number Theory Phys., 1:2 (2007), 347-452; arXiv: math-ph/0702045; L. Chekhov, B. Eynard, JHEP, 03 (2006), 014; arXiv: hep-th/0504116; 12 (2006), 026; arXiv: math-ph/0604014; B. Eynard, N. Orantin, M. Marinño, JHEP, 06 (2007), 058; arXiv: hep-th/0702110; N. Orantin, From matrix models' topological expansion to topological string theories: counting surfaces with algebraic geometry, arXiv: 0709.2992; Gaussian matrix model in an external field and non-intersecting Brownian motions, arXiv: 0803.0705. 
[13] B. Eynard, JHEP, 01 (2003), 051; 11 (2003), 018; arXiv: hep-th/0210047; arXiv: hep-th/0309036.

[14] M. Bertola, B. Eynard, J. Phys. A, 36:28 (2003), 7733-7750; arXiv: hep-th/0303161; B. Eynard, A short note about Morozov's formula, arXiv: math-ph/0406063; B. Eynard, A. Prats Ferrer, Comm. Math. Phys., 264:1 (2005), 115-144; arXiv: hep-th/0502041; A. Prats Ferrer, B. Eynard, P. Di Francesco, J.-B. Zuber, J. Stat. Phys., 129:5-6 (2009), 885-935; arXiv: math-ph/0610049; M. Bertola, A. Prats Ferrer, Int. Math. Res. Not., 16 (2008), Art. ID rnn062; arXiv:0801.3452; M. Bergère, B. Eynard, J. Phys. A, 42:26 (2009), 265201; arXiv: 0805.4482.

[15] N.A. Nekrasov, A. Okounkov, "Seiberg-Witten theory and random partitions", The Unity of Mathematics, Progr. Math., 244, eds. P. Etingof, V. Retakh, I. M. Singer, Birkhäuser Boston, Boston, MA, 2006, 525-596; arXiv: hep-th/0306238; A. Marshakov, N. A. Nekrasov, JHEP, 01 (2007), 104; arXiv: hep-th/0612019; B. Eynard, J. Stat. Mech. Theory Exp., 7 (2008), P07023; arXiv: 0804.0381; A. Klemm, P. Sułkowski, Nucl. Phys. B, 819:3 (2009), 400-430; arXiv: 0810.4944; P. Sulkowski, Phys. Rev. D, 2009, 086006; arXiv: 0904.3064.

[16] А.С. Александров, А.Д. Миронов, А. Ю. Морозов, ТМФ, 150:2 (2007), 179-192; arXiv: hep-th/0605171; Physica D, 235:1-2 (2007), 126-167; arXiv: hep-th/0608228; N. Orantin, Symplectic invariants, Virasoro constraints and Givental decomposition, arXiv: 0808.0635 .

[17] J. Harer, D. Zagier, Invent. Math., 85:3 (1986), 457-485; S. K. Lando, A. K. Zvonkine, Graphs on Surfaces and Their Applications, Encyclopaedia Math. Sci., 141, Springer, Berlin, 2004; E. T. Akhmedov, Sh. Shakirov, Gluing of surfaces with polygonal boundaries, arXiv: 0712.2448; A. Morozov, Sh. Shakirov, JHEP, 12 (2009), 003; arXiv: 0906.0036; "Harer-Zagier correlation functions in Gaussian matrix models", to appear.

[18] A. Morozov, Sh. Shakirov, Combinatorial Solution of Hermitian Model at Low Genera, to appear.

[19] A. Gerasimov, A. Marshakov, A. Mironov, A. Morozov, A. Orlov, Nucl. Phys. B, 357:2-3 (1991), 565-618; Yu. Makeenko, A. Marshakov, A. Mironov, A. Morozov, Nucl. Phys. B, 356:3 (1991), 574-628; S. Kharchev, A. Marshakov, A. Mironov, A. Morozov, S. Pakuliak, Nucl. Phys. B, 404:3 (1993), 717-750; arXiv: hep-th/9208044.

[20] М. Л. Концевич, Функи. анализ и его прил., 25:2 (1991), 50-57; M. Kontsevich, Comm. Math. Phys., 147:1 (1992), 1-23.

[21] S. Kharchev, A. Marshakov, A. Mironov, A. Morozov, A. Zabrodin, Phys. Lett. B, 275:3-4 (1992), 311-314; arXiv: hep-th/9111037; Nucl. Phys. B, 380:1-2 (1992), 181-240; arXiv: hep-th/9201013; S. Kharchev, A. Marshakov, A. Mironov, and A. Morozov, Nucl. Phys. B, 397:1-2 (1993), 339-378; arXiv: hep-th/9203043; A. Marshakov, A. Mironov, A. Morozov, Modern Phys. Lett. A, 7:15 (1992), 1345-1359; arXiv: hep-th/9201010; Phys. Lett. B, 274:3-4 (1992), 280-288; arXiv: hep-th/9201011.

[22] M. Adler, P. van Moerbeke, Comm. Math. Phys., 147 (1992), 25-56; P. Di Francesco, C. Itzykson, J.-B. Zuber, Comm. Math. Phys., 151:1 (1993), 193-219; arXiv: hep-th/9206090; S. Kharchev, A. Marshakov, A. Mironov, A. Morozov, Modern Phys. Lett. A, 8:11 (1993), 1047-1061; С. М. Харчев, А. В. Маршаков, А.Д. Миронов, А. Ю. Морозов, ТМФ, 95:2 (1993), 280-292; arXiv: hep-th/9208046.

[23] M. Fukuma, H. Kawai, R. Nakayama, Internat. J. Modern Phys. A, 6:8 (1991), 1385-1406; R. Dijkgraaf, E. Verlinde, H. Verlinde, Nucl. Phys. B, 348:3 (1991), 435-456; A. Mironov, A. Morozov, Phys. Lett. B, 252:1 (1990), 47-52; F. David, Modern Phys. Lett. A, 5:13 (1990), 1019-1029; J. Ambjørn, Yu. Makeenko, Modern Phys. Lett. A, 5:22 (1990), 1753-1763; H. Itoyama, Y. Matsuo, Phys. Lett. B, 255:2 (1991), 202-208.

[24] L. Chekhov, Yu. Makeenko, Phys. Lett. B, 278:3 (1992), 271-278; arXiv: hep-th/9202006; Modern Phys. Lett. A, 7:14 (1992), 1223-1236; arXiv: hep-th/9201033; J. Ambjørn, 
C. F. Kristjansen, Yu. M. Makeenko, Modern Phys. Lett. A, 7:34 (1992), 3187-3202; arXiv: hep-th/9207020.

[25] E. J. Martinec, Comm. Math. Phys., 138:3 (1991), 437-449; V. Periwal, D. Shevitz, Phys. Rev. Lett., 64:12 (1990), 1326-1329; Nucl. Phys. B, 344:3 (1990), 731-746; K. Demeterfi, C.-I. Tan, Modern Phys. Lett. A, 5:20 (1990), 1563-1574.

[26] M. J. Bowick, A. Morozov, D. Shevitz, Nucl. Phys. B, 354:2-3 (1991), 496-530.

[27] V.A. Kazakov, A. A. Migdal, Nucl. Phys. B, 397:1-2 (1993), 214-238; arXiv: hep-th/9206015.

[28] M. Caselle, A. D'Adda, S. Panzeri, Phys. Lett. B, 293:1-2 (1992), 161-167; arXiv: hep-th/9207086; 302:1 (1993), 80-86; arXiv: hep-th/9212074.

[29] I. I. Kogan, A. Morozov, G. W. Semenoff, N. Weiss, Nucl. Phys. B, 395:3 (1993), 547-580; arXiv: hep-th/9208012; Internat. J. Modern Phys. A, 8:8 (1993), 1411-1436; arXiv: hep-th/9208054; M. Dobroliubov, A. Morozov, G. W. Semenoff, N. Weiss, Internat. J. Modern Phys. A, 9:29 (1994), 5033-5051; arXiv: hep-th/9312145.

[30] A. Morozov, Modern Phys. Lett. A, 7:37 (1992), 3503-3507; arXiv: hep-th/9209074.

[31] S. L. Shatashvili, Comm. Math. Phys., 154:2 (1993), 421-432; arXiv: hep-th/9209083.

[32] S. Kharchev, A. Marshakov, A. Mironov, A. Morozov, Internat. J. Modern Phys. A, 10:14 (1995), 2015-2051; arXiv: hep-th/9312210.

[33] A. Mironov, A. Morozov, G. W. Semenoff, Internat. J. Modern Phys. A, 11:28 (1996), 5031-5080; arXiv: hep-th/9404005.

[34] A. Hurwitz, Math. Ann., 39:1 (1891), 1-60; 55:1 (1901), 53-66.

[35] R. Dijkgraaf, "Mirror symmetry and elliptic curves", The Moduli Spaces of Curves, Progr. Math., 129, eds. R. Dijkgraaf, C. Faber G. van der Geer, Brikhäuser, Boston, MA, 1995, 149-163; R. Vakil, Enumerative geometry of curves via degeneration methods, Harvard Ph. D. Thesis, 1997; I. P. Goulden, D. M. Jackson, Proc. Amer. Math. Soc., 125:1 (1997), 51-60; arXiv:math/9903094; Д. Звонкин, С. К. Ландо, Функи. анализ и его прил., 33:3 (1999), 21-34; S. Lando, D. Zvonkine, Mosc. Math. J., 7:1 (2007), 85-107; arXiv: math.AG/0303218; S. Natanzon, V. Turaev, Topology, 38:4 (1999), 889-914; I. P. Goulden, D. M. Jackson, A. Vainshtein, Ann. Comb., 4:1 (2000), 27-46; A. Okounkov, Math. Rev. Lett., 7:4 (2000), 447-453; arXiv: math/0004128; A. B. Givental, Mosc. Math. J., 1:4 (2001), 551-568; arXiv: math/0108100; T. Ekedahl, S. Lando, M. Shapiro, A. Vainshtein, Invent. Math., 146:2 (2001), 297-327; C. К. Ландо, УМH, 57:3(345) (2002), 29-98; A. V. Alexeevski, S. M. Natanzon, Selecta Math., 12:3-4 (2006), 307-377; arXiv: math.GT/0202164; "Hurwitz numbers for regular coverings of surfaces by seamed surfaces and Cardy-Frobenius algebras of finite groups", Geometry, Topology, and Mathematical Physics, Amer. Math. Soc. Transl. Ser. 2, 224, eds. V. M. Buchstaber, I. M. Krichever, AMS, Providence, RI, 2008, 1-25; А.В. Алексеевский, С. М. Натанзон, Изв. РАН. Сер. матем., 72:4 (2008), 3-24; А. В. Алексеевский, С. М. Натанзон, УМН, 61:4(370) (2006), 185-186; S. M. Natanzon, Disk single Hurwitz numbers, arXiv: 0804.0242; J. Zhou, Hodge integrals, Hurwitz numbers and symmetric groups, arXiv: math. AG/0308024; A. Okounkov, R. Pandharipande, Ann. Math., 163:2 (2006), 517-560; arXiv: math.AG/0204305; T. Graber, R. Vakil, Compos. Math., 135:1 (2003), 25-36; М.Э. Казарян, С. К. Ландо, Изв. РАН. Сер. матем., 68:5 (2004), 91-122; arXiv:math.AG/0410388; An algebro-geometric proof of Witten's conjecture, arXiv: math/0601760; M. Kazarian, Adv. Math., 221:1 (2009), 1-21; arXiv: 0809.3263; S. Lando, "Combinatorial Facets of Hurwitz numbers", Applications of Group Theory to Combinatorics, eds. J. Koolen, J. H. Kwak, M.-Y. Xu, CRC Press, Boca Raton, FL, 2008, 109-132; V. Bouchard, M. Mariño, "Hurwitz numbers, matrix models and enumerative geometry", From Hodge Theory to Integrability and TQFT: $t t^{*}$-geometry, Proc. Sympos. Pure Math., 78, eds. R. Y. Donagi, K. Wendland, AMS, Providence, RI, 2008, 263-283; arXiv: 0709.1458; A. Mironov, A. Morozov, JHEP, 02 (2009), 024; arXiv: 0807.2843. 
[36] A. Morozov, Sh. Shakirov, JHEP, 04 (2009), 064; arXiv: 0902.2627.

[37] G. Borot, B. Eynard, M. Mulase, B. Safnuk, A matrix model for simple Hurwitz numbers and topological recursion, arXiv: 0906.1206; A. Morozov, Sh. Shakirov, On equivalence of two Hurwitz matrix models, arXiv: 0906.2573.

[38] A. Mironov, A. Morozov, S. Natanzon, Complete set of cut-and-join operators in Hurwitz-Kontsevich theory, arXiv: 0904.4227.

[39] A. Mironov, A. Morozov, S. Natanzon, Integrability and $\mathcal{N}$-point Hurwitz numbers, to appear.

[40] A. Alexandrov, A. Mironov, A. Morozov, Cut-and-Join operators, matrix models and characters, to appear.

[41] E. Brezin, D. J. Gross, Phys. Lett. B, 97:1 (1980), 120-124; D. J. Gross, E. Witten, Phys. Rev. D, 21:2 (1980), 446-453.

[42] A. Alexandrov, A. Mironov, A. Morozov, BGWM as the second constituent of complex matrix model, arXiv: 0906.3305.

[43] Harish-Chandra, Amer. J. Math., 79:1 (1957), 87-120; 80:2 (1958), 241-310.

[44] C. Itzykson, J.-B. Zuber, J. Math. Phys., 21:3 (1980), 411-421; P. Zinn-Justin, J.-B. Zuber, J. Phys. A, 36:12 (2003), 3173-3193; arXiv: math-ph/0209019.

[45] B. De Wit, G.'t Hooft, Phys. Lett. B, 69:1 (1977), 61-64.

[46] A. B. Givental, Int. Math. Res. Not., 2001, no 23 (2001), 1265-1286; arXiv: math. AG/0008067.

[47] H. Leutwyler, A. Smilga, Phys. Rev. D, 46:12 (1992), 5607-5632; J. J. M. Verbaarschot, Universal behavior in Dirac spectra, arXiv: hep-th/9710114; R. Brower, P. Rossi, C.-I. Tan, Nucl. Phys. B, 190:4 (1981), 699-718; T. Akuzawa, M. Wadati, J. Phys. Soc. Japan, 67:7 (1998), 2151-2154.

[48] G. Frobenius, Berlin Akad., Wiss., 1896, 985-1021; Ф.Г. Фробениус, Теория характеров и представлений групn, КомКнига, M., 2005; D. E. Littlewood, The theory of group characters and matrix representations of groups, Clarendon Press, Oxford, 1950; F. Murnaghan, The Theory of Group Representations, Dover, New York, 1963; М. Хамермеш, Теория групп и ее применение к физическим проблемам, Мир, М., 1966; И. Макдональд, Симметрические функции и многочлены Холла, Мир, М., 1984; В. Simon, Representations of Finite and Compact Groups, Grad. Stud. Math., 10, AMS, Providence, 1996; W. Fulton, Young Tableaux. With applications to representation theory and geometry, London Math. Soc. Stud. Texts, 35, Cambridge Univ. Press, Cambridge, 1997; B. Sagan, The Symmetric Group. Representations, Combinatorial Algorithms, and Symmetric Functions, Grad. Texts Math., 203, Springer, New York, 2001.

[49] A. B. Balantekin, J. Math. Phys., 25:6 (1984), 2028-2030; Phys. Rev. D, 62:8 (2000), 085017; arXiv: hep-th/0007161.

[50] М. А. Семенов-Тян-Шанский, Изв. АН СССР. Сер. матем., 40:3 (1976), 562-592; J. J. Duistermaat, G. J. Heckman, Invent. Math., 69:2 (1982), 259-268; 72:1 (1983), 153-158; A. Alekseev, L. Faddeev, S. Shatashvili, J. Geom. Phys., 5:3 (1988), 391-406; M. Blau, E. Keski-Vakkuri, A. Niemi, Phys. Lett. B, 246:1-2 (1990), 92-98; E. Keski-Vakkuri, A. Niemi, G. Semenoff, O. Tirkkonen, Phys. Rev. D, 44:12 (1991), 3899-3905; A. Hietamäki, A. Yu. Morozov, A. J. Niemi, K. Palo, Phys. Lett. B, 263:3-4 (1991), 417-424; A. Yu. Morozov, A. J. Niemi, K. Palo, Phys. Lett. B, 271:3-4 (1991), 365-371; Nucl. Phys. B, 377:1-2 (1992), 295-338; R. J. Szabo, Equivariant Localization of Path Integrals, arXiv: hep-th/9608068; Y. Karshon, Lecture Notes on Group Action on Manifolds, 1996-1997; M. Stone, Nucl. Phys. B, 314:3 (1989), 557-586.

[51] A. Losev, N. Nekrasov, S. Shatashvili, Nucl. Phys. B, 534:3 (1998), 549-611; arXiv: hep-th/9711108; The Freckled instantons, arXiv: hep-th/9908204; Class. Quant. Grav., 17:5 (2000), 1181-1187; arXiv: hep-th/9911099; N. A. Nekrasov, Adv. Theor. Math. Phys., 7:5 (2003), 831-864; arXiv: hep-th/0206161; R. Flume, R. Poghossian, Internat. 
J. Modern Phys. A, 18:14 (2003), 2541-2563; arXiv: hep-th/0208176; E. Frenkel, A. Losev, N. Nekrasov, Nucl. Phys. Proc. Suppl., 171 (2007), 215-230; arXiv: hep-th/0702137.

[52] N. Seiberg, E. Witten, Nucl. Phys. B, 426:1 (1994), 19-52; Erratum, 430 (1994), 485-486; arXiv: hep-th/9407087; A. Gorsky, I. M. Krichever, A. Marshakov, A. Mironov, A. Morozov, Phys. Lett. B, 355:3-4 (1995), 466-474; arXiv: hep-th/9505035; R. Donagi, E. Witten, Nucl. Phys. B, 460:2 (1996), 299-334; arXiv: hep-th/9510101; E. J. Martinec, Phys. Lett. B, 367:1-4 (1996), 91-96; arXiv: hep-th/9510204; A. Gorsky, A. Marshakov, Phys. Lett. B, 375:1-4 (1996), 127-134; arXiv: hep-th/9510224; H. Itoyama, A. Morozov, Nucl. Phys. B, 477:3 (1996), 855-877; arXiv: hep-th/9601168; 491:3 (1997), 529-573; arXiv: hep-th/9512161; N. Nekrasov, Nucl. Phys. B, 531:1-3 (1998), 323-344; arXiv: hep-th/9609219; A. Marshakov, A. Mironov, A. Morozov, Phys. Lett. B, 389:1 (1996), 43-52; arXiv: hep-th/9607109; Modern Phys. Lett. A, 12:11 (1997), 773-787; arXiv: hep-th/9701014; Internat. J. Modern Phys. A, 15:8 (2000), 1157-1206; arXiv: hep-th/9701123; H.W. Braden, A. Marshakov, A. Mironov, A. Morozov, Phys. Lett. B, 448:3-4 (1999), 195-202; arXiv: hep-th/9812078; Nucl. Phys. B, 558:1-2 (1999), 371-390; arXiv: hep-th/9902205; Acta Appl. Math., 99:3 (2007), 223-244; arXiv: hep-th/0606035; A. Gorsky, A. Marshakov, A. Mironov, A. Morozov, Phys. Lett. B, 380:1-2 (1996), 75-80; A note on spectral curve for the periodic homogeneous $X Y Z$-spin chain, arXiv: hep-th/9604078; Nucl. Phys. B, 527:3 (1998), 690-716; arXiv: hep-th/9802007.

[53] F. Calogero, J. Math. Phys., 10:12 (1969), 2191-2196; 12:3 (1971), 419-436; C. F. Dunkl, Trans. Amer. Math. Soc., 311:1 (1989), 167-183; Canad. J. Math., 43:6 (1991), 1213-1227; Н. Ю. Решетихин, Л.А. Тахтаджян, Л. Д. Фаддеев, Алгебра и анализ, 1:1 (1989), 178-206; A. Alekseev, S. Shatashvili, Nucl. Phys. B, 323:3 (1989), 719-733; A. Gorsky, N. Nekrasov, Nucl. Phys. B, 414:1-2 (1994), 213-238; arXiv: hep-th/9304047; 436:3 (1995), 582-608; arXiv: hep-th/9401017; P. Etingof, I. B. Frenkel, A. A. Kirillov Jr., Duke Math. J., 80:1 (1995), 59-90; arXiv: hep-th/9407047; A. Gerasimov, S. Kharchev, A. Morozov, M. Olshanetsky, A. Marshakov, A. Mironov, Internat. J. Modern Phys. A, 12:14 (1997), 2523-2583; arXiv: hep-th/9601161; P. Etingof, Lectures on Calogero-Moser systems, arXiv: math/0606233.

[54] V.V. Dolotin, On discriminants of polylinear forms, arXiv: alg-geom/9511010; A. Morozov, Sh. Shakirov, JHEP, 12 (2009), 002; arXiv: 0903.2595; K. Fujii, Beyond Gaussian: a comment, arXiv: 0905.1363.

[55] I. M. Gel'fand, M.M. Kapranov, A.V. Zelevinsky, Discriminants, Resultants and Multidimensional Determinants, Math. Theory Appl., Birkhäuser, Boston, MA, 1994; V. Dolotin, A. Morozov, Introduction to Non-Linear Algebra, World Sci., Hackensack, NJ, 2007; arXiv: hep-th/0609022; The Universal Mandelbrot Set. Beginning of the Story, World Sci., Hackensack, NJ, 2006; Algebraic Geometry of Discrete Dynamics. The case of one variable, arXiv: hep-th/0501235; Internat. J. Modern Phys. A, 23:22 (2008), 3613-3684; arXiv: hep-th/0701234; E. T. Akhmedov, V. Dolotin, A. Morozov, Писвма в ЖЭЭТФ, 81:12 (2005), 776-779; arXiv: hep-th/0504160; А. Ю. Морозов, М. Н. Сербин, ТМФ, 154:2 (2008), 316-343; V. Dolotin, A. Morozov, Sh. Shakirov, A-infinity-structure on simplicial complexes, arXiv:0704.2609; Phys. Lett. B, 651:1 (2007), 71-73; arXiv: 0704.2884; A. Morozov, Писъма в ЖЭЭТФ, 86:11 (2007), 856-859; A. Morozov, Sh. Shakirov, Analogue of the identity Log Det=Trace Log for resultants, arXiv:0804.4632; Resultants and contour integrals, arXiv:0807.4539; А. С. Анохина, А. Ю. Морозов, Ш.Р. Шакиров, ТМФ, 160:3 (2009), 403-433; arXiv: 0812.5013.

[56] Y. Nambu, Phys. Rev. D, 7:8 (1973), 2405-2412; В. Т. Филиппов, Сиб. матем. журн., 26:6 (1985), 126-140; L. Takhtajan, Comm. Math. Phys., 160:2 (1994), 295-315; arXiv: hep-th/9301111; J. Hoppe, Helv. Phys. Acta, 70:1-2 (1997), 302-317; arXiv: 
hep-th/9602020; H. Awata, M. Li, D. Minic, T. Yoneya, JHEP, 02 (2001), 013; arXiv: hep-th/9906248; J. Schwarz, JHEP, 11 (2004), 078; arXiv: hep-th/0411077; A. Basu, J. A. Harvey, Nucl. Phys. B, 713:1-3 (2005), 136-150; arXiv: hep-th/0412310; J. Bagger, N. Lambert, Phys. Rev. D, 75:4 (2007), 045020; arXiv: hep-th/0611108; 77:6 (2008), 065008; arXiv: 0711.0955; 79:2 (2009), 025002; arXiv:0807.0163; JHEP, 02 (2008), 105; arXiv:0712.3738; A. Gustavsson, Nucl. Phys. B, 811:1-2 (2009), 66-76; arXiv: 0709.1260; JHEP, 04 (2008), 083; arXiv: 0802.3456; S. Mukhi, C. Papageorgakis, JHEP, 5 (2008), 085; arXiv: 0803.3218; M. A. Bandres, A. E. Lipstein, J. H. Schwarz, JHEP, 05 (2008), 025; arXiv: 0803.3242; A. Morozov, JHEP, 05 (2008), 076; arXiv: 0804.0913; Писъма в ЖКЭТФ, 87:12 (2008), 759-762; arXiv: 0805.1703; J. Gomis, G. Milanesi, J. G. Russo, JHEP, 06 (2008), 075; arXiv: 0805.1012; S. Benvenuti, D. Rodríguez-Gómez, E. Tonni, H. Verlinde, JHEP, 01 (2009), 078; arXiv: 0805.1087; P.-M. Ho, Y. Imamura, Y. Matsuo, JHEP, 07 (2008), 003; arXiv: 0805.1202; O. Aharony, O. Bergman, D. L. Jafferis, J. Maldacena, JHEP, 10 (2008), 091; arXiv: 0806.1218; I. A. Bandos, P. K. Townsend, Class. Quant. Grav., 25:24 (2008), 245003; arXiv: 0806.4777; JHEP, 02 (2009), 013; arXiv: 0808.1583; J. Gomis, D. Rodíguez-Gómez, M. Van Raamsdonk, H. Verlinde, JHEP, 09 (2008), 113; arXiv: 0807.1074; J. A. Minahan, W. Schulgin, K. Zarembo, JHEP, 03 (2009), 057; arXiv: 0901.1142; J. Figueroa-O'Farrill, Deformations of 3-algebras, arXiv: 0903.4871; D. Kamani, Evidence for the $p+1$-algebra for super-p-brane, arXiv: 0904.2721.

[57] B. Schlittgen, T. Wettig, J. Phys. A, 36:12 (2003), 3195-3201; arXiv: math-ph/0209030.

[58] C. Lehner, T. Wettig, T. Guhr, Y. Wei, J. Math. Phys., 49:6 (2008), 063510; arXiv: 0801.1226.

[59] Ф.А. Березин, Ф. И. Карпелевич, ДАН СССР, 118:1 (1958), 9-12; Т. Guhr, Т. Wettig, J. Math. Phys., 37:12 (1996), 6395-6413; A. D. Jackson, M. K. Şener, J. J. M. Verbaarschot, Phys. Lett. B, 387:2 (1996), 355-360.

[60] R. Dijkgraaf, E. Witten, Comm. Math. Phys., 129:2 (1990), 393-429.

[61] А. Ю. Морозов, УФН, 162:8 (1992), 83-175.

[62] M. Jimbo, T. Miwa, Publ. RIMS Kyoto Univ., 19:3 (1983), 943-1001; K. Ueno, K. Takasaki, "Toda lattice hierarchy", Group Representations and Systems of Differential Equations, Adv. Stud. Pure Math., 4, ed. K. Okamoto, North-Holland, Amsterdam, 1984, 1-95; A. Morozov, L. Vinet, Internat. J. Modern Phys. A, 13:10 (1998), 1651-1707; arXiv: hep-th/9409093; А. Д. Миронов, А. Ю. Морозов, Л. Винэ, ТМФ, 100:1 (1994), 119-131; arXiv: hep-th/9312213; A. Gerasimov, S. Khoroshkin, D. Lebedev, A. Mironov, A. Morozov, Internat. J. Modern Phys. A, 10:18 (1995), 2589-2614; arXiv: hep-th/9405011; S. Kharchev, A. Mironov, A. Morozov, TMФ, 104:1 (1995), 129-143; arXiv: q-alg/9501013; A. Mironov, "Quantum deformations of $\tau$-functions, bilinear identities and representation theory", Symmetries and Integrability of Difference Equations, CRM Proc. Lecture Notes, 9, eds. D. Levi, L. Vinet, P. Winternitz, AMS, Providence, RI, 1996, 219-237; arXiv: hep-th/9409190; А. Д. Миронов, ТМФ, 114:2 (1998), 163-232; arXiv: q-alg/9711006. 